\title{
Localization of MHD and fast particle modes using reflectometry in ASDEX Upgrade
}

\author{
S da Graça ${ }^{1}$, G D Conway ${ }^{2}, \mathrm{P}$ Lauber $^{2}$, M Maraschek ${ }^{2}$, \\ D Borba ${ }^{1}$, S Günter ${ }^{2}$, L Cupido ${ }^{1}$, K Sassenberg ${ }^{3}$, F Serra ${ }^{1}$, \\ ME Manso ${ }^{1}$, the CFN reflectometry group ${ }^{1}$ and the \\ ASDEX Upgrade Team ${ }^{2}$ \\ ${ }^{1}$ Centro de Fusão Nuclear CFN-IST, Associação EURATOM-IST, Portugal \\ 2 Max-Planck-Institut für Plasmaphysik, EURATOM-Association IPP, D-85748 \\ Garching, Germany \\ 3 Physics Department, University College Cork, Cork, Ireland
}

\begin{abstract}
The radial structure of Toroidal Alfvén Eigenmodes (TAEs) is of great importance for comparison with theoretical predictions. A dual-channel fast frequency hopping millimeter-wave reflectometer installed on the ASDEX Upgrade tokamak is capable of measuring density fluctuations from the plasma edge to core, allowing the radial eigenfunction of $n=4$ TAE and edge MHD modes to be obtained using phase perturbation and coherence data analysis techniques. The two techniques reveal similar results, and in particular the radial structure of the $n=4$ TAE is found to be in good agreement with numerical predictions from linear gyrokinetic simulations. First results of the radial localization of Alfvén cascades are also presented.
\end{abstract}

Submitted to: Plasma Phys. Control. Fusion

PACS numbers: 52.55.Fa, 52.35.Ra, 52.70.Gw 


\section{Introduction}

The physics of energetic particle driven modes is of particular interest for understanding fast ion dynamics and transport in burning plasma devices, such as ITER [1, 2]. Alfvén Eigenmodes (AE) instabilities, for example, can expel alpha particles, potentially damaging the first wall components of a reactor as well as reducing the efficiency of plasma self-heating. AE instabilities such as Toroidicity induced Alfvén Eigenmodes (TAEs) and Alfvén Cascades (ACs) have been shown to be destabilized by Neutral Beam Injection (NBI) [2] and Ion Cyclotron Resonance Heating (ICRH) [4]. TAEs modes have been observed in many devices such as TFTR [2], DIII-D [5], NSTX [6], Alcator C-mod [7], JT60U [4], JET [8] and ASDEX Upgrade [9].

An analysis of the toroidal and poloidal mode numbers, as well as the radial extent of TAEs is of great importance for the validation of theoretical predictions, which can be used to determine the behaviour of fast particle modes in ITER. Many diagnostic techniques have been used to investigate the radial eigenfunction of TAEs and ACs. In TFTR, the TAE radial eigenfunction was obtained for the first time using Beam Emission Spectroscopy (BES) and reflectometry. The poloidal mode number was found to be consistent within $50 \%$ of the predictions from an ideal MHD model [10]. Nazikian et al. investigated the radial mode structure of a $n=4$ TAE using three fixed frequency X-mode reflectometer channels. Radial scanning was achieved by varying the toroidal magnetic field strength $\left(B_{t}\right)[11]$. However, several discharges with identical magnetic activity were required to obtain the radial scans across the core region. The inferred density fluctuation $\left(\delta n_{e} / n_{e}\right)$ profile showed a ballooning structure in qualitative agreement with ideal MHD model predictions. Recently, the $n=2$ TAE with a core localized anti-ballooning structure has been re-interpreted as an AC [12].

In Tore Supra, Sabot et al. measured the $\delta n_{e} / n_{e}$ radial profile of a TAE mode using $\mathrm{X}$-mode reflectometry in a single discharge. The mode exhibited a core localized peak towards the high-field-side (HFS), however, no detailed comparisons with theoretical models were made [13].

In DIII-D, Carolipio et al. measured the radial mode structure of an $n=5 \mathrm{TAE}$ using soft X-ray diagnostics, but poor agreement was found with ideal MHD (NOVA), gyrofluid (TAE/FL) and global gyrokinetic wave (PENN) models. In ASDEX Upgrade, Borba et al. compared the emissivity fluctuation amplitude of soft X-ray channels in the core region with simulation, it was found that $n=3$ TAE is global [9].

Recently, Electron Cyclotron Emission (ECE) diagnostics have revealed good potential for studying the radial mode structure of TAEs [14]. In DIII-D the radial eigenfunction obtained with ECE on the low-field-side shows good agreement with ideal MHD modelling for $n=3 \mathrm{TAE}$ and $n=3$ AC modes [15]. Other diagnostics measuring density fluctuations through different vertical or radial chords, such as two colour $\mathrm{CO}_{2}$ interferometry [16], far-infrared scattering (FIR) [17] and Phase Contrast Imaging (PCI) on Alcator C-mod [18] have been used to obtain information on the localization of Alfvén Eigenmodes. 
On ASDEX Upgrade a heterodyne fast frequency hopping reflectometry has been developed to measure density fluctuation profiles with a fast $(\sim 100 \mathrm{~ms})$ time scale. In this paper, first measurements of complete radial scans from edge to core in a single discharge with phase and amplitude are presented. Two data analysis techniques for extracting the radial eigenfunction, reflectometer phase perturbation and reflectometermagnetics coherence, are compared. The results show both core and edge localized peaks, which are shown to be in good agreement with numerical predictions from the linear gyrokinetic simulation code LIGKA [19]. An additional edge localized mode has also been observed in TAE discharges but has not yet been conclusively identified. Finally, first results on the localization and characteristics of ACs in ASDEX Upgrade are presented.

\section{Diagnostic hardware}

Plasma reflectometry is based on the radar principle: a microwave beam with a frequency $F_{0}$ is launched into the plasma, parallel to the density gradient, and is reflected when the refractive index approaches zero. For an O-mode polarized beam, the cutoff condition (in the cold plasma approximation) depends only on the electron density. The cutoff density is given by:

$$
n_{c}=\frac{m_{e} \epsilon_{0}\left(2 \pi F_{0}\right)^{2}}{e^{2}}
$$

where $e$ the electron charge, $m_{e}$ the electron mass and $\epsilon_{0}$ the vacuum permittivity.

In this study two O-mode reflectometer systems have been used on ASDEX Upgrade: a multi-channel LFS/HFS (Low Field Side/ High Field Side) broadband FM-CW (Frequency Modulation- Continuous Wave) profile reflectometer capable of operation in either swept or fixed frequency mode, and a dual channel fast frequency hopping reflectometer launching from the LFS which is dedicated to density fluctuation measurements.

Figure 1(a) shows a schematic of one channel of the fast frequency hopping reflectometer [20]. Each channel uses two phase locked programmable frequency synthesizers (8 - $12 \mathrm{GHz}$ ) for heterodyne receiving, followed by passive and active frequency multipliers to bring the launch frequency up to $32-49.2 \mathrm{GHz}$ (Q-band) and $49-72 \mathrm{GHz}$ (V-band) respectively. These frequency ranges correspond to the density range $1.35-6 \times 10^{19} \mathrm{~m}^{-3}$. Each channel/band uses an individual single antenna (with invessel directional coupler for transmit and receive separation) located approximatively at the tokamak midplane and tilted poloidally to follow the vessel wall. Figure 2 shows a poloidal cross-section of the tokamak with flux surfaces for a lower single null discharge illustrating the antenna positions and vacuum lines-of-sight. The lines of sight are aligned to be as close to perpendicular to the density cutoff layers in the plasma edge region as possible. Both channels are equipped with In-phase $I=A(t) \cos (\varphi(t))$ and Quadrature $Q=A(t) \sin (\varphi(t))$ detection allowing separation of the phase $\varphi(t)$ and amplitude $A(t)$ fluctuation signals. The data acquisition system currently has 12-bit 


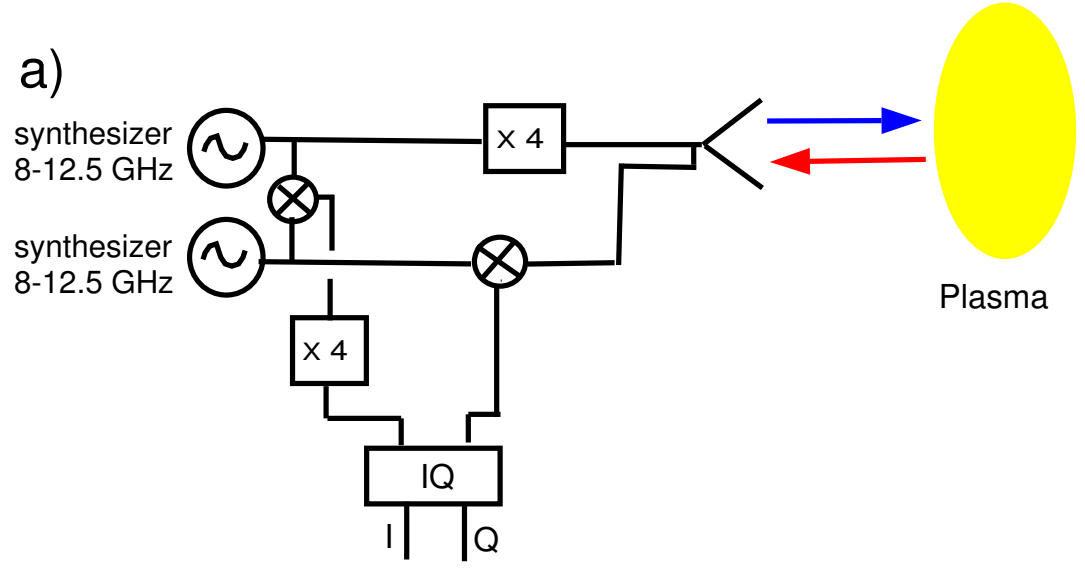

b)

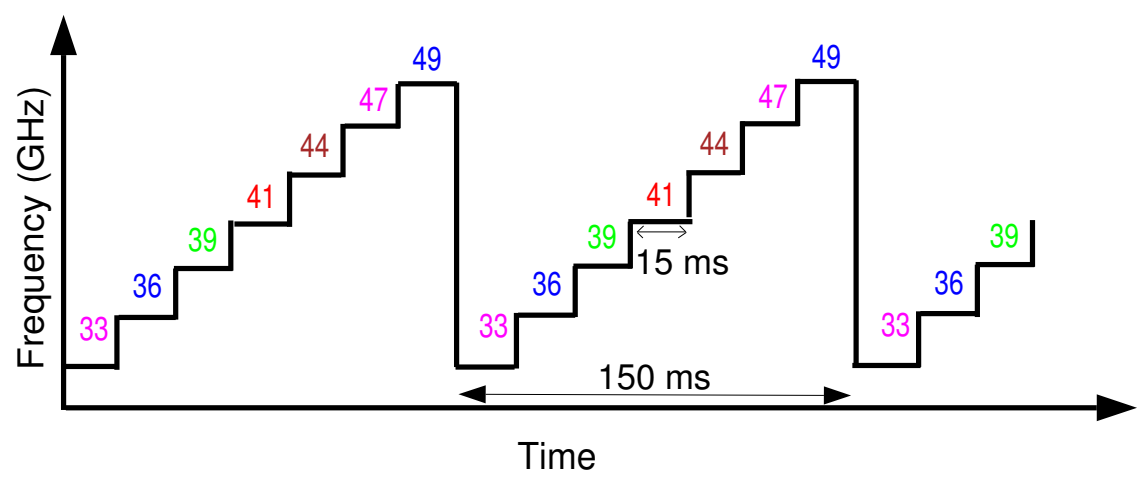

Figure 1. Schematic of measurement technique: (a) Heterodyne reflectometer with IQ detection, (b) Typical staircase launch frequency pattern.

resolution with a $1 \mathrm{MHz}$ sample rate, which permits discrimination of MHD modes and turbulent density fluctuations up to $500 \mathrm{kHz}$. Each channel can be pre-programmed with a specified launch frequency pattern. Figure 1(b) shows a typical staircase frequency pattern which is repeated continuously throughout a single plasma discharge. The probe frequency is typically held constant for at least $6 \mathrm{~ms}$ and can change and lock to another frequency in less than $0.8-1.2 \mathrm{~ms}$ - hence the name "fast frequency hopping reflectometer".

Figure 3 shows a toroidal view of the ASDEX Upgrade tokamak. The fast frequency hopping reflectometer antennas are located between toroidal sectors 4 and 5 , while the FM-CW profile reflectometer (REF) antennas are sited toroidally between sectors 5 and 6. Also shown are the positions of the main Mirnov coil arrays and other relevant plasma density diagnostics, such as the Thomson scattering (TS) system in sector 3, the Lithium beam (LID) diagnostic in sector 9 and interferometers (DCN) in section 11.

The profile reflectometer [21] covers the frequency range $16-100 \mathrm{GHz}$ in several bands $\left(n_{e}=0.3-10 \times 10^{19} \mathrm{~m}^{-3}\right)$ on the LFS and $16-72 \mathrm{GHz}\left(n_{e}=0.3-6.64 \times 10^{19} \mathrm{~m}^{-3}\right)$ on the HFS. Density profiles can be obtained with a high temporal resolution of $35 \mu \mathrm{s}$ and high spatial resolution of $5 \mathrm{~mm}$. When operated in fixed frequency mode 


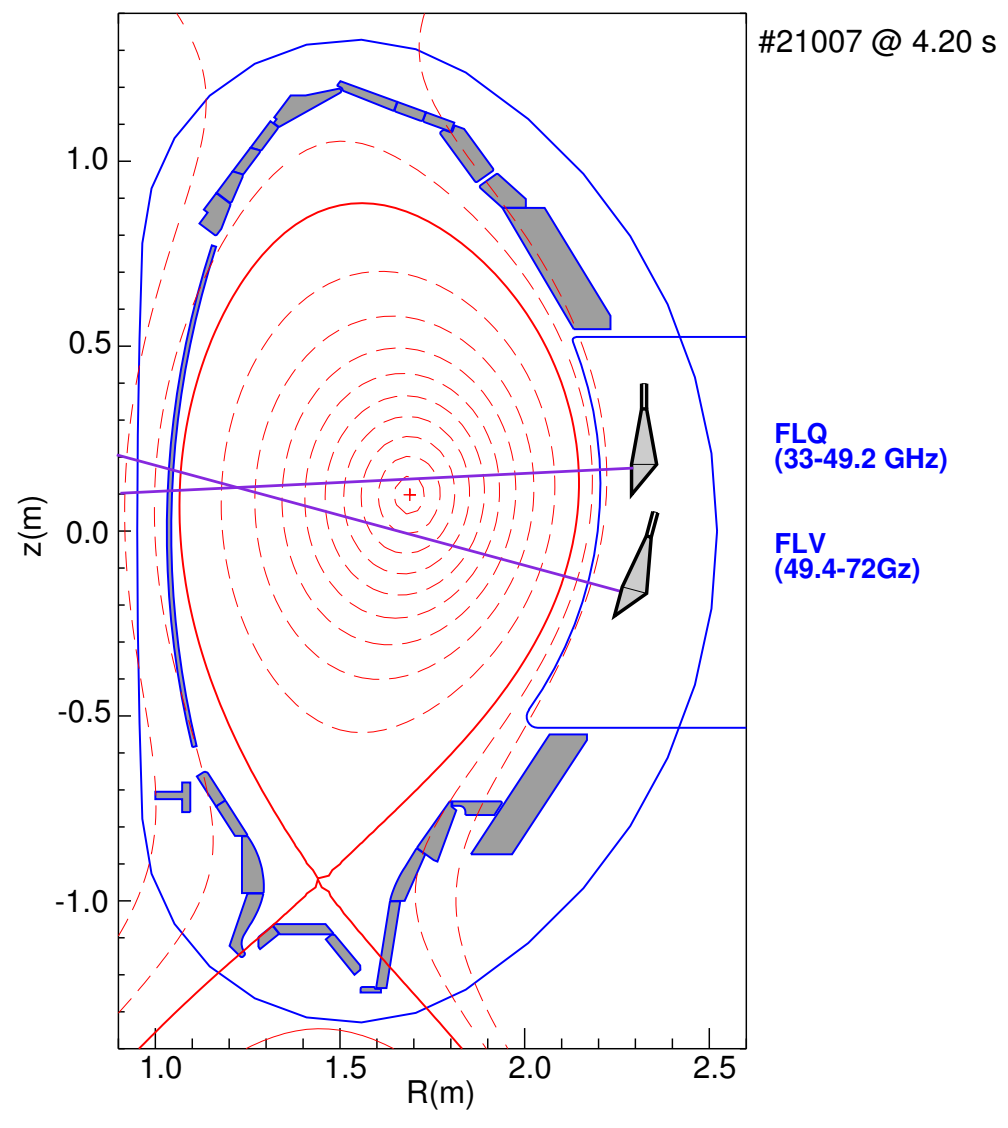

Figure 2. Poloidal position of reflectometry system.

simultaneous fluctuation measurements from 4 radial positions can be obtained, but only with qualitative information due to the limitation of the single ended homodyne detection method used.

\section{Data analysis methods}

\subsection{Correlation and coherence analysis.}

Cross correlation and coherence techniques are widely used data analysis tools for studying the temporal and spatial structure of fluctuation phenomena. For example, in fusion plasmas the coherence between reflectometry or Electron Cyclotron Emission (ECE) and magnetic signals has been used to radially localize MHD activity as a means of providing information on the current (or $q$ ) profile [22]. They are also employed with radial and poloidal correlation reflectometers (e.g. [23, 24]) where two spatially separated reflectometry fluctuation signals are correlated to obtain the size of coherent structures.

The time delayed cross correlation coefficient between two digitized zero mean signals $x(t)$ and $y(t)$ (normalized cross correlation function) is defined as

$$
\rho_{x y}(\tau)=\frac{R_{x y}(\tau)}{\left(R_{x x}(0) R_{y y}(0)\right)^{1 / 2}}
$$




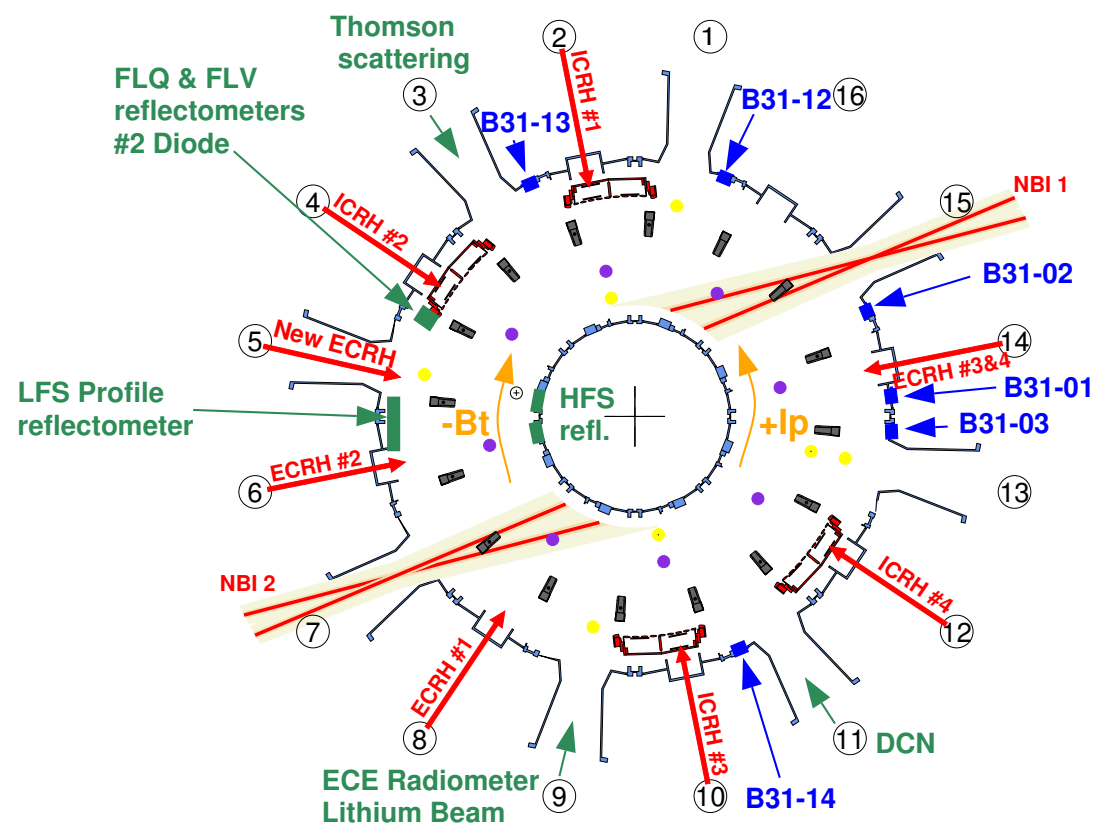

Figure 3. Toroidal position of reflectometry systems and Mirnov coils (blue).

where $\tau$ is a variable time delay and $R_{i j}$ the respective sample cross and auto correlation functions [25]

$$
R_{x y}(\tau)=\frac{1}{N} \sum_{q=1}^{N} x\left(t_{q}\right) y\left(t_{q}+\tau\right),
$$

where time $t_{q}=q \Delta t$ with $\Delta t$ the sample interval. The correlation coefficient ranges between \pm 1 and is unity when the $x(t)$ and $y(t)$ signals are identical. In the frequency domain the coherence spectrum $\gamma(f)$ is simply the Fourier transform of $\rho_{x y}(\tau)$. The coherence is normally computed directly from the FFTs of the $x(t)$ and $y(t)$ signals [26] and is defined as

$$
\gamma(f)=\frac{<S_{x y}(f)>}{\left[<S_{x x}(f)>\cdot<S_{y y}(f)>\right]^{1 / 2}}
$$

where $S_{x y}$ is the cross-power spectrum and $S_{x x}, S_{y y}$ the auto-power spectra. The brackets $<>$ represent ensemble averages. The cross-power spectrum is defined as the sum of co $\left(C_{x y}\right)$ and quad $\left(Q_{x y}\right)$ spectra [26]

$$
S_{x y}(f)=\left[C_{x y}(f)^{2}+Q_{x y}(f)^{2}\right]^{1 / 2} .
$$

The relative spectral phase angle between signals $x(t)$ and $y(t)$ is given by the cross-phase spectrum $\theta_{x y}(f)$

$$
\theta_{x y}(f)=\tan ^{-1}\left(\frac{Q_{x y}(f)}{C_{x y}(f)}\right)
$$

which corresponds to the time delay $\Gamma$ between two signals as a function of frequency

$$
\Gamma(f)=\frac{\theta_{x y}(f)}{2 \pi f} .
$$


The coherence is a statistical parameter obtained by ensemble averaging the crosspower spectrum over many data sets. The number of data sets $N_{a v}$ defines the minimum level of statistical significance in the coherence $\gamma_{o}=1 / \sqrt{N_{a v}}$, if data sets are statistically independent. Here, the data set duration is $512 \mu \mathrm{s}$ while the autocorrelation time of both reflectometer and magnetic signals is around $2 \mu \mathrm{s}$. Due to the limitation of the signal lengths there is always a compromise between minimizing the statistical noise level (number of averages) and maximizing the frequency resolution in the spectra (number of FFT points).

In the study performed here the radial structure of coherent oscillations such as MHD and fast particles modes was obtained by correlating the reflectometer signal (typically the in-phase homodyne signal) for each launch frequency with the corresponding magnetic fluctuation signal from the nearest toroidally located Mirnov coil to give the coherence of the mode versus radial position. Investigations revealed similar results for the phase, amplitude, In-phase, Quadrature and complex amplitude signals [27]. For each radial position 10 to $15 \mathrm{~ms}$ of data are used. FFT data lengths are typically 512 points with a $50 \%$ overlap sampling [25]. While the coherence is normally quite stable for each cutoff layer measurement it is necessary to monitor the temporal evolution of the cross-phase as it may drift significantly over the measurement period. For these measurements the standard deviation as well as the mean cross-phase are calculated.

\subsection{Phase perturbation method.}

The I-Q detection of the hopping frequency reflectometer allows the separation of the phase $\varphi(t)$ and amplitude $A(t)$ fluctuations. The phase signal is sensitive to the radial displacement of density cutoff layer, as well as interference effects, while the amplitude signal is dominated by scattering effects. The phase signal can be expressed as $\varphi(t)=\varphi_{0}+\delta \varphi(t)$ with mean phase $\varphi_{0}$ related to the position of the cutoff layer and $\delta \varphi(t)$ the phase fluctuation near the cutoff layer. The relation between $\varphi$ and the density fluctuation level $\delta n_{e} / n_{e}$ has been extensively studied. Generally, for small scale plasma turbulence sophisticated 2D models are required, such as [28]. However, for large scale plasma perturbations, such as low order MHD modes, where the transverse fluctuation wavelength $\Lambda$ is much larger than the radius $w$ of the microwave probing beam $(\Lambda \gg w)$, a simpler 1D model can be used such as $[29,30,31]$. Here, the density fluctuation level is given by [31],

$$
\delta \varphi=\frac{4 \pi}{\lambda} \frac{\delta n_{e}}{n_{e}} L_{n}
$$

where $L_{n}=n_{c} /\left(\nabla n_{e}\right)$ is the density gradient length at the cutoff density, $\lambda$ is the vacuum wavelength of the probing wave. Strictly speaking, this relation is only valid if $\left(\delta n_{e} / n_{e}\right) \leq\left(k_{r} L_{n}\right)^{-1}$ (referred to as the mixing length limit [32]) i.e small amplitude fluctuations with radial plasma fluctuation wavenumbers $k_{r}$ much smaller than the 
wavenumber of the probing wave. This formula has been used to estimate the density fluctuation level induced by MHD activity like magnetic islands [33].

Experimentally, the algorithm of Seo [34] is used to obtain $\varphi(t)$. It calculates the relative phase differences between two time points and then accumulates them to give the absolute phase. This method is valid if the phase variation between successive measurements is within $\pm \pi$. To study the influence of MHD activity on density fluctuations the reflectometry signal is normally band-pass filtered for the frequencies of interest and then the standard deviation calculated. The disadvantage of this procedure is that modes with small frequency separation can not be isolated due to the finite response of the filter and in addition the effect of any background turbulence present in this frequency band can not be separated. Some authors have proposed using the magnitude of the spectral peak at the mode frequency from the FFT of the phase signal $[11,33]$. Here, we calculate the maximum amplitude of $\varphi(t)$ at frequency $f$ by integrating the phase spectrum across the mode frequency peak, subtracting the spectral power from adjacent frequency bands to compensate for background turbulence effects, then multiplying by 2 to consider negative and positive frequencies.

To convert the reflectometer phase modulation to a density fluctuation level requires a separate measurement of the density profile. This is also needed to give the radial cutoff position for each reflectometer probing frequency. A best fitted density profile is obtained using a combination of TS, LID and REF data when available. The normalized poloidal flux radius $\rho_{\text {pol }}$ is normally used as the radial coordinate as it accounts for the different diagnostic lines of sight, and is defined from the tokamak scrape-off-layer (SOL) to the core. The error bars in reflectometer density cutoff layer position are based on the quality of the density profile. These values include the resolutions of the respective diagnostics, the density profile fitting errors, as well as the temporal variation of the density data during the measurement interval.

\section{Overview of Alfvén Eigenmodes}

In a tokamak, an MHD instability can be generally characterized by its poloidal $m$ and toroidal $n$ mode numbers, its frequency of rotation $\omega$, and the radial displacement of magnetic field lines $\zeta_{0}$

$$
\zeta=\zeta_{0}(r) \exp (-i m \theta+i n \phi-i \omega t)
$$

where $\theta$ and $\phi$ are the geometric poloidal and toroidal angles respectively (toroidal effects are not considered in this ansatz [35]).

In tokamaks, the toroidal geometry breaks the continuum Alfvén spectra and permits discrete toroidal Alfvén Eigenmodes (TAEs) with frequencies inside the continuum gaps [36]. In toroidal plasmas the dispersion relation for Alfvén Eigenmodes is locally given by

$$
f_{a}(r)=\frac{v_{a}}{(2 \pi R)}(n-m / q(r)),
$$




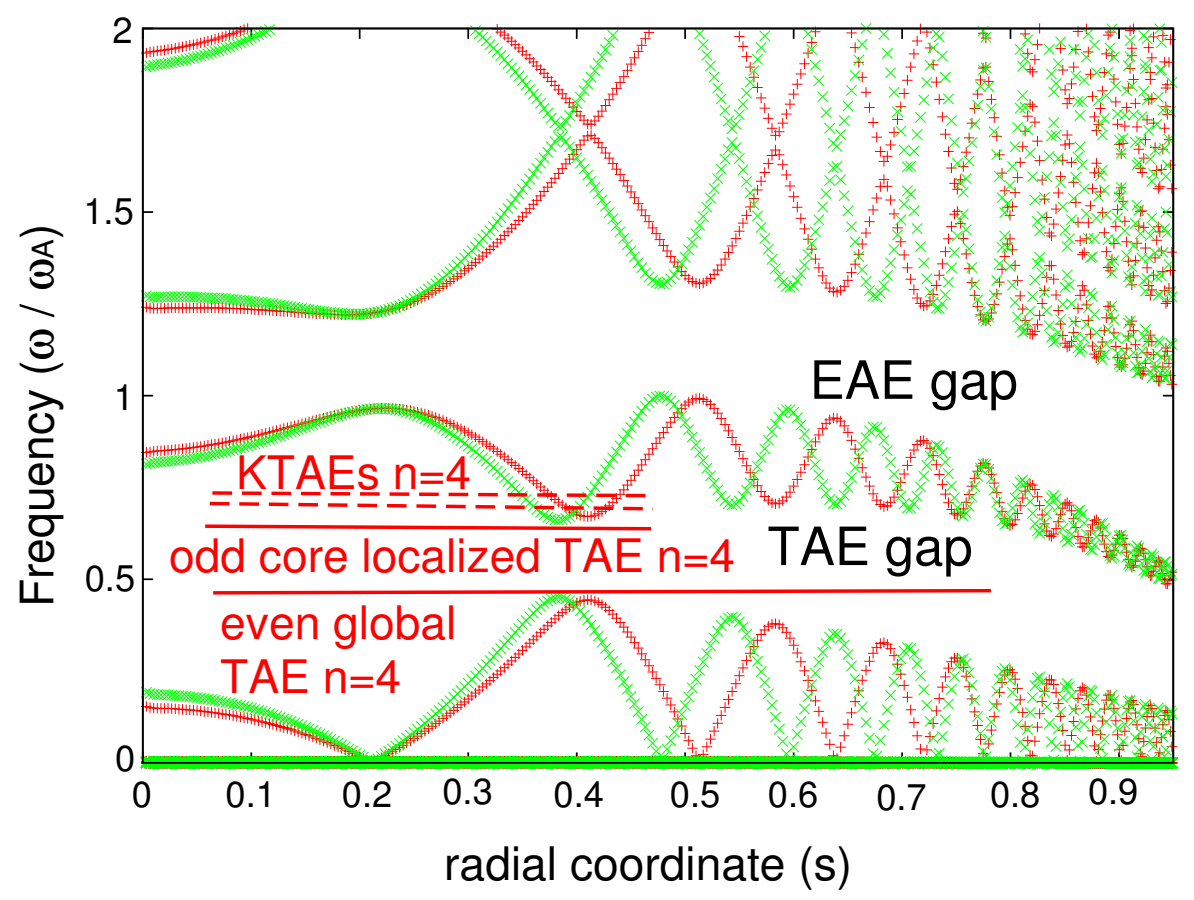

Figure 4. Alfvén continuum of $n=4$ (red) and $n=5$ (green) for shot \#20398 at $t=1.76 \mathrm{~s}$.

where $v_{a}$ is the Alfvén velocity [37]

$$
v_{a}=B / \sqrt{4 \pi \rho}
$$

and $R$ the major radius of the tokamak, $q(r)$ the safety factor, $B$ the equilibrium magnetic field and $\rho$ the plasma mass density. The coupling of two poloidal harmonics $m$ and $m+1$ produces the TAE gap frequency with

$$
f_{\mathrm{TAE}}=v_{a} /(4 \pi q R) .
$$

Figure 4 shows an Alfvén continuum spectra computed from the CASTOR code [38] for discharge \#20398. Two types of TAEs (odd and even) can exist depending on the mode frequency. The even TAE has a frequency just above the lower bound of the corresponding continuum gap and shows a ballooning structure [39]. The odd TAE is peaked in amplitude on HFS and its frequency is near the upper continuum of the TAE gap $[40,41]$. Depending on the TAE radial structure, two different kinds of TAE exist - core localized TAEs and global TAEs. The core localized TAE exists at low magnetic shear in the core and is located in a single continuum gap. The global TAE crosses several continuum gaps with a radial mode structure extended to the plasma edge [42].

The kinetic toroidicity induced Alfvén Eigenmodes (KTAEs) with a frequency just above the TAE gap are generated by the coupling of two kinetic Alfvén waves (KAWs) [43]. The KTAE's radial structure is given by LIGKA code. 


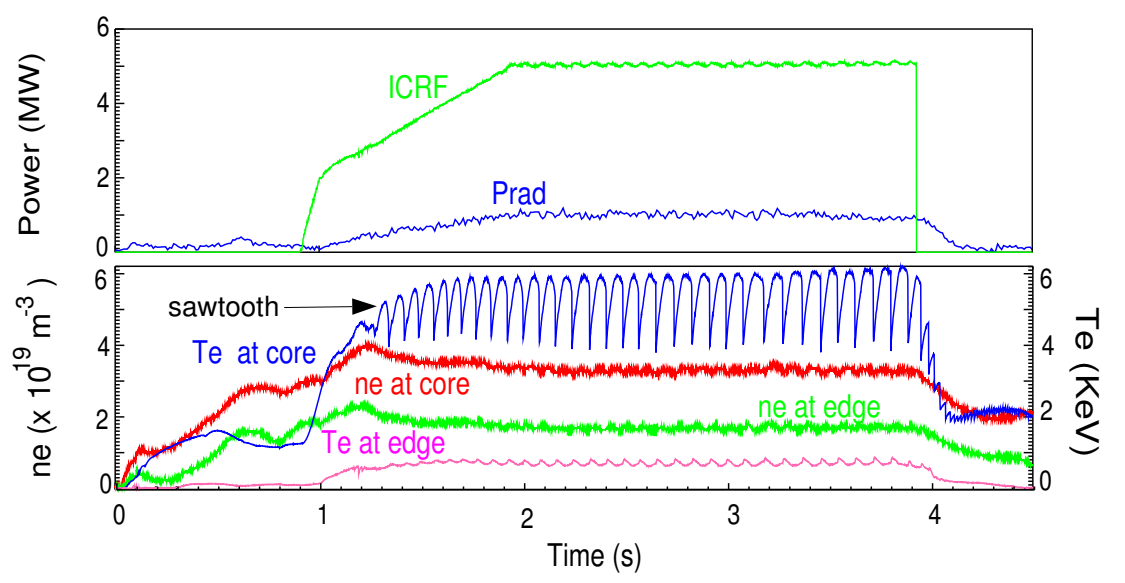

Figure 5. Time traces of several plasma parameters for shot \#20489.

Alfvén Cascades (ACs) were discovered in JT60U [44] and are associated with the temporal evolution of $q_{\min }(t)$ with a frequency ramp rate described by

$$
\frac{d}{d t} \omega_{\mathrm{AC}} \approx \frac{m v_{a}}{R} \frac{d}{d t} q_{\mathrm{min}}^{-1}(t)
$$

Generally ACs are observed in tokamak discharges with a nearly flat or reversed $q$ profile $[45,46,12,47]$. Hence these modes are of particular interest in the study of internal transport barrier (ITB) formation since they can be used to indicate the presence of magnetic shear reversal [48]. ACs exhibit upward frequency sweeping, starting at a frequency that has been interpreted as being close to the geodesic curvature frequency [49] and rises towards the TAE gap. According to the theory of Fu and Berk [50] the reason why the cascade frequency occasionally cannot penetrate into the TAE gap may be due to the background pressure gradient, as observed in JET experimental data. Depending on the gap structure, also ACs coupling to the continuum - resulting in stronger damping - can occur [51].

In DIII-D, Beta induced Alfvén Eigenmodes (BAEs) are observed with intense neutral beam injection in high beta $(\beta)$ plasma discharges [52]. BAE frequencies are lower than the TAE frequency and vary with $B_{t}$, and are predicted to be excited only in the plasma core. A possible explanation for the BAE behaviour is that the BAE frequency gap is caused by coupling between an Alfvén wave and a sound wave due to $\beta$ effects [53].

\section{Toroidal Alfvén Eigenmodes}

\subsection{TAE discharge}

In ASDEX Upgrade, unstable TAEs are observed in magnetic probe, reflectometer, and soft X-ray camera signals in low density, $n_{e} \approx 3-5 \times 10^{19} \mathrm{~m}^{-3}$, discharges with ion cyclotron resonance heating $(\mathrm{ICRH})$ power exceeding $P_{\mathrm{ICRH}}>2.5 \mathrm{MW}[9]$. In this density range the frequency hopping reflectometers can probe cutoff layers from 


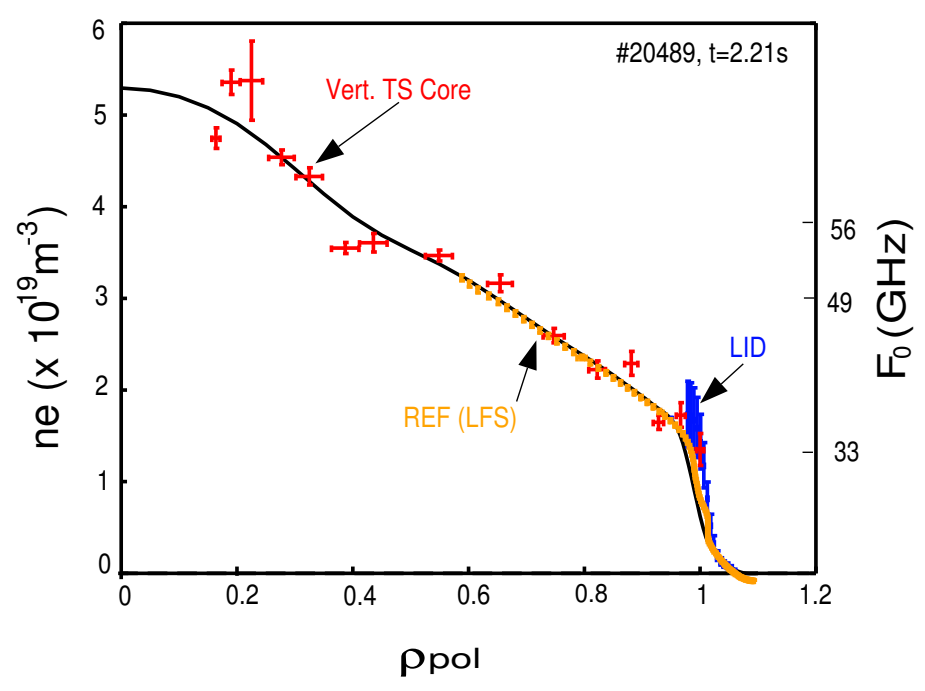

Figure 6. Spline fitted density profile (solid line) using Thomson scattering (TS), Lithium beam (LID) and FM-CW reflectometry (REF) data for shot \#20489 at $2.21 \mathrm{~s}$.

the edge to the core, i.e. $1.35-6.64 \times 10^{19} \mathrm{~m}^{-3}$, for discharges with a reasonably steep density profile. Figure 7 shows time-frequency spectrograms from (a) In-phase Q-band reflectometer and (b) magnetic signals for the typical discharge \#20489 with $5.5 \mathrm{MW} \mathrm{ICRH,} B_{T}=-2.5 \mathrm{~T}, I_{p}=0.8 \mathrm{MA}$ and $\bar{n}_{e} \approx 3.4 \times 10^{19} \mathrm{~m}^{-3}$. Figure 5 shows the corresponding time traces of ICRH power, radiated power, density and electron temperature for this shot, while figure 6 shows the density profile at 2.21 seconds.

The Mirnov spectrogram in figure 7 clearly shows a set of TAE modes around $230 \mathrm{kHz}$, interrupted by sawteeth events roughly every $80 \mathrm{~ms}$. After each sawtooth a downward frequency sweeping mode with harmonics appears around $100 \mathrm{kHz}$. During the time frame shown the Q-band hopping frequency reflectometer makes two and a half profile sweeps. The TAEs are only seen in the 47 and $49 \mathrm{GHz}$ steps (and also at higher frequencies in the V-band reflectometer data - which is not shown here) confirming the TAEs are primarily localized to the core region. The lower frequency modes appear only in the $33-39 \mathrm{GHz}$ steps indicating they are edge localized.

From a mode analysis of Mirnov coil data toroidal mode numbers of $n=3,4,5,6$ rotating in ion diamagnetic drift direction are deduced for the TAEs. Combining the $\mathrm{Q}$ and $\mathrm{V}$-band reflectometer measurements only TAEs with $n=4,5,6$ are observed with a radial extension of $\rho_{\text {pol }} \approx 0.4-0.7( \pm 0.05)$. Concerning the $n=3 \mathrm{TAE}$, it is either observed at the edge $\left(\rho_{\text {pol }}>0.97\right)$ or in the core $\left(\rho_{\text {pol }}<0.4\right)$. In the magnetic measurements after each sawtooth crash there is a clear reduction in the TAE amplitude, particularly for the $n=3$ mode, which is believed to be due to the removal of fast particles from the core thus removing the drive of TAEs [9]. The different behaviour of the $n=3$ TAE mode may be explained by the different localization of the mode. Figure 7(a) shows a broad frequency striation (e.g. at $t=2.08 \mathrm{~s}$ and $\rho_{\text {pol }} \simeq 0.7$ ) in the reflectometer data coinciding with the onset of the sawtooth. This is due to the rapid radial movement of the density cutoff layer as a consequence of the particle expulsion 


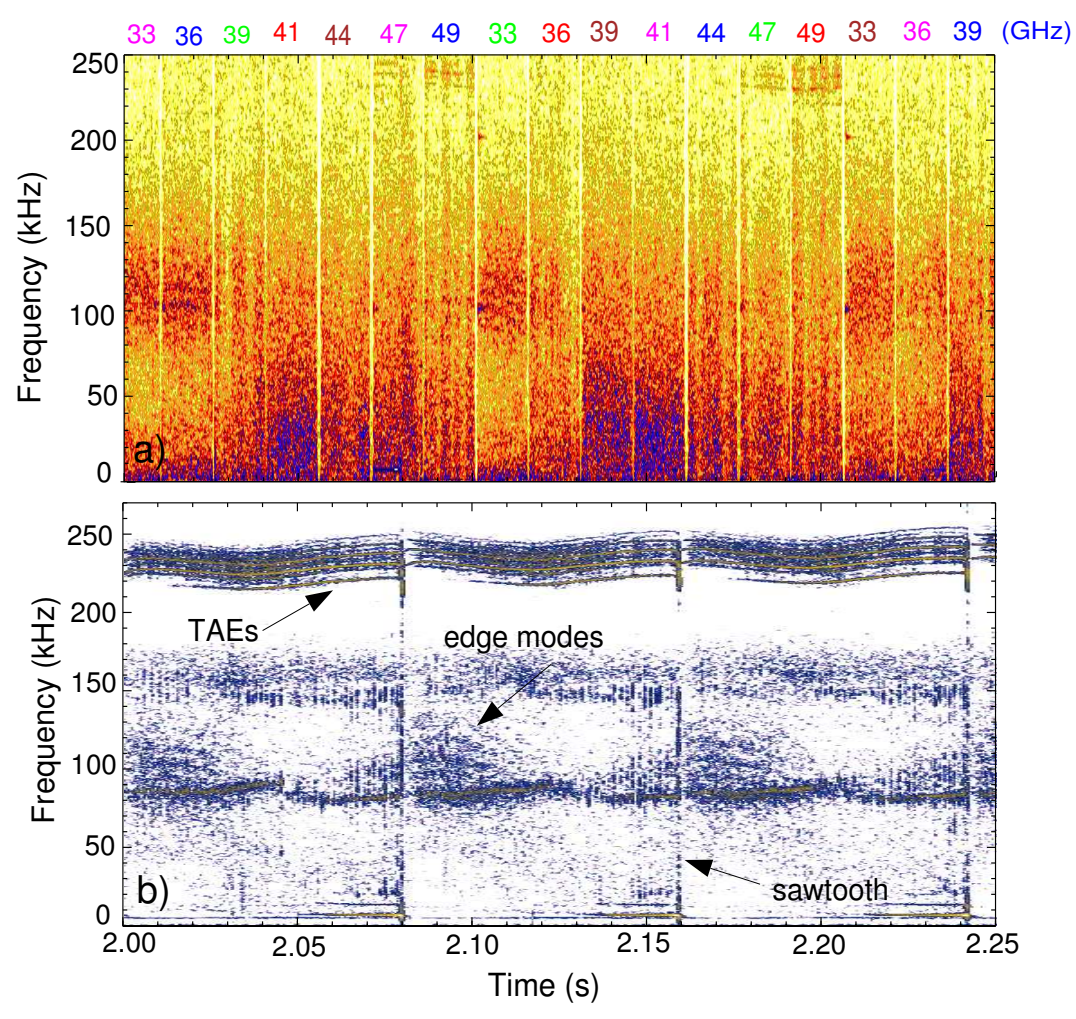

Figure 7. Time-frequency spectrograms of (a) FLQ-I reflectometer and (b) Mirnov coil signals for shot \#20489.

from the core [54]. In the magnetic spectrogram, figure 7(b), the TAE modes just stop at each sawtooth crash.

In summary, the reflectometry measurements give information on the localization of the various MHD phenomena: MHD modes at the plasma edge after each sawtooth, the sawtooth and its precursor, as well as TAEs in the core. The radial position and extent of these modes are observed to be almost constant from successive reflectometer sweeps. Figures 8 and 9 show power spectra for edge $(33 \mathrm{GHz})$ and core $(49 \mathrm{GHz})$ reflectometer cutoff layers together with a Mirnov signal $(\dot{\mathrm{B}}(\mathrm{t}))$ spectra. The low frequency turbulence $(f<50 \mathrm{kHz})$ generally dominates in all the reflectometer signals, but the relative amplitude of the edge modes and TAEs can be compared with the magnetics. The internal localized reflectometer and the external magnetic coil measurements explain the different relative amplitude between TAEs. Also observed in reflectometer data are small amplitude spectral peaks (sidebands) around the main $n=4$ and $n=5$ TAE peaks which may possibly be identified as nonlinear splitting of the TAE spectral line $[55]$.

\subsection{Phase analysis}

To demonstrate the phase analysis technique, discharge \#21007 with 4.5 MW pure $\mathrm{ICRH}, B_{T}=-2 \mathrm{~T}, I_{p}=1 \mathrm{MA}$ and $\bar{n}_{e} \approx 3.6 \times 10^{19} \mathrm{~m}^{-3}$ was used. From magnetic measurements TAE modes with $n=4,5,6$ are present, although the $n=6$ mode is 


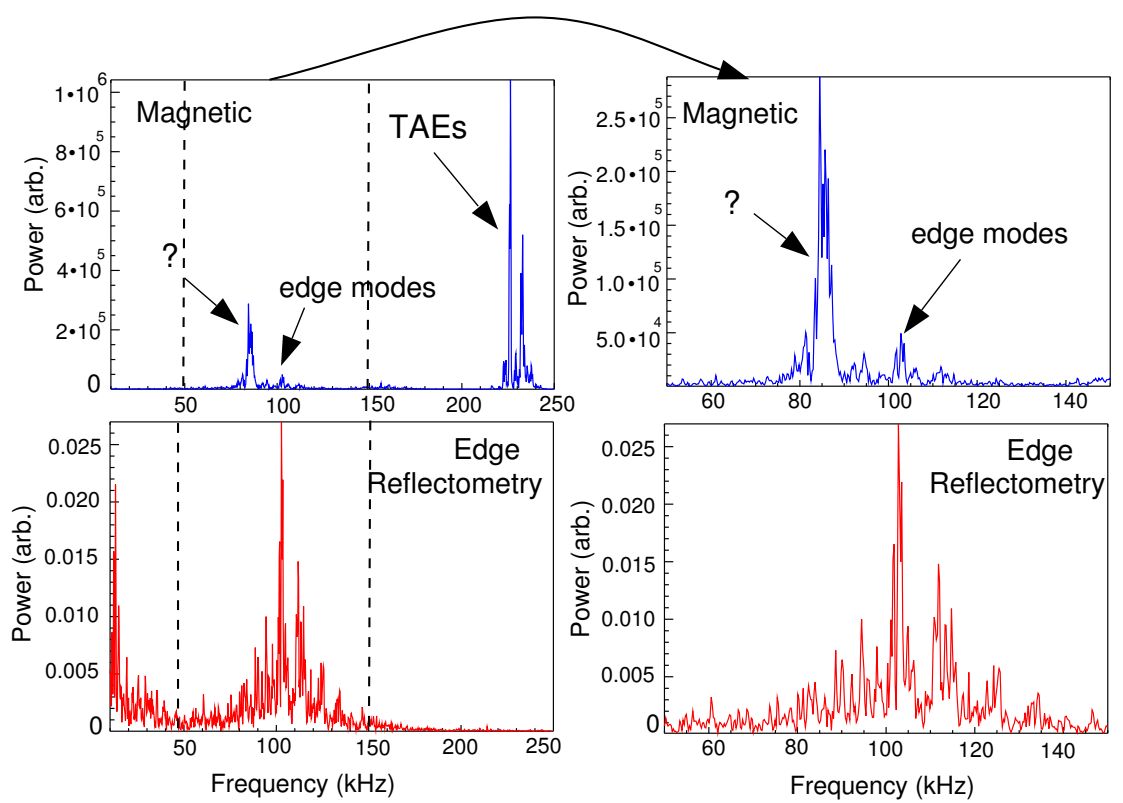

Figure 8. Magnetic and FLQ-I spectrum at $t=2.013-2.023 \mathrm{~s}$ (33 GHz edge) for shot \#20489. Magnetic spectra show edge MHD modes at around $100 \mathrm{kHz}$, TAEs at around $220 \mathrm{kHz}$ and unidentified modes at around $85 \mathrm{kHz}$. Edge reflectometer spectra show only edge MHD modes.
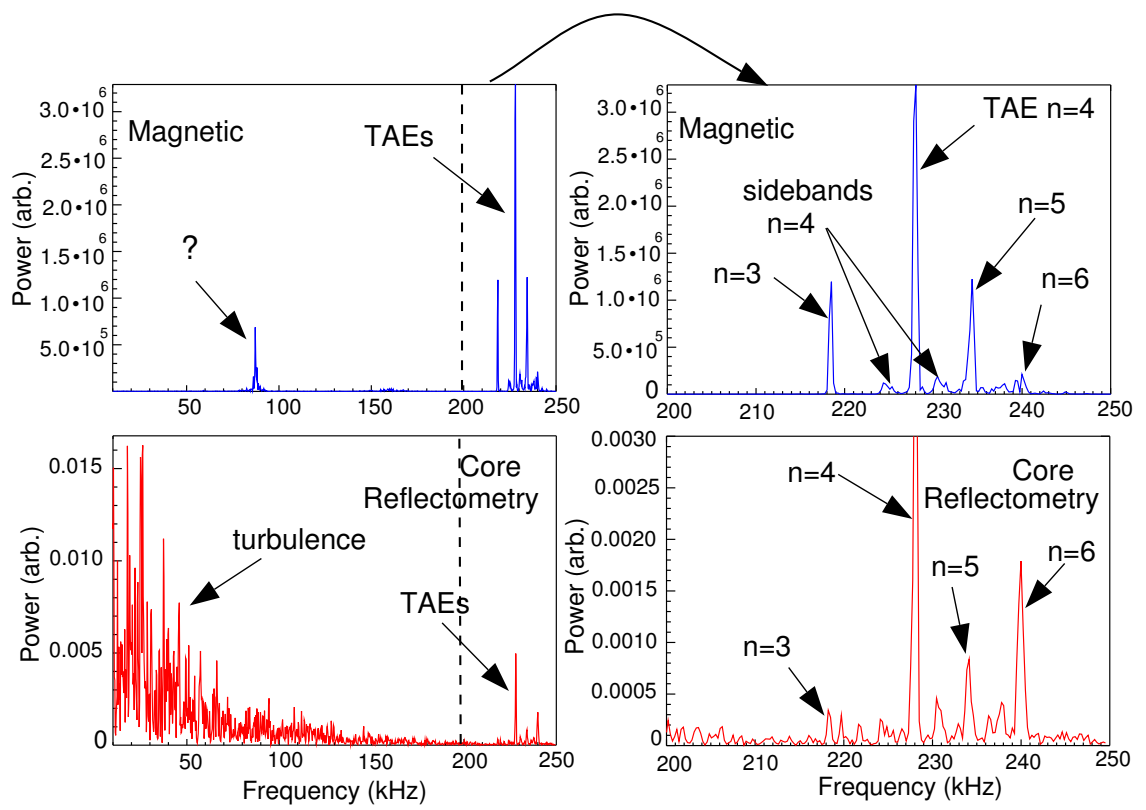

Figure 9. Magnetic and FLQ-I spectrum at $t=2.193-2.2 \mathrm{~s}$ (49 GHz core) for shot \#20489. Magnetic spectra show again TAEs and other unidentified modes at around $85 \mathrm{kHz}$. Core reflectometer spectra show TAEs and turbulence. 


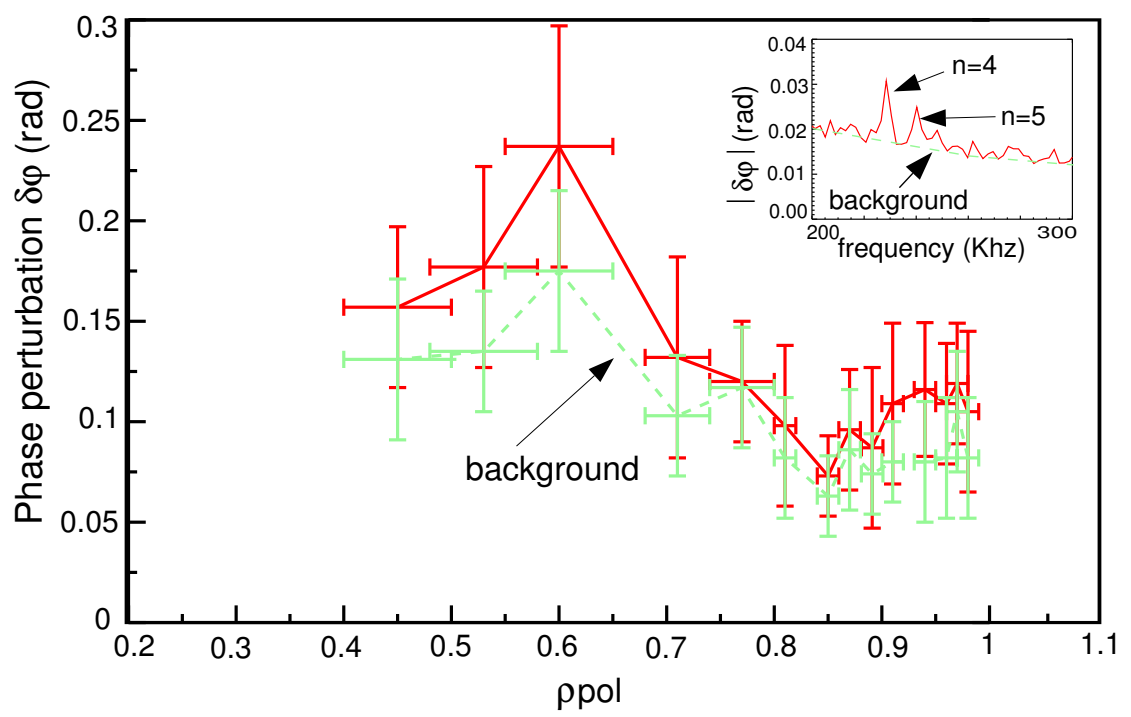

Figure 10. Reflectometer phase perturbation profile for $n=4$ TAE mode, background profile and phase spectra (insert) for shot \#21007.

intermittent, appearing just after each sawtooth and disappearing after around $200 \mathrm{~ms}$. The reflectometer frequency pattern ( $\mathrm{Q}$ and $\mathrm{V}$-bands) is 11 steps of $15 \mathrm{~ms}$ covering density fluctuations from the edge $\left(\rho_{\text {pol }}=0.97 \pm 0.01\right)$ to the core $\left(\rho_{\text {pol }}=0.45 \pm 0.05\right)$. For this discharge the reflectometry spectrograms clearly show TAE modes present at both the core and the edge, however, to assess their relative strength requires a more quantitative analysis.

In the reflectometer phase spectrum at least two peaks are observed at around $230 \mathrm{kHz}(n=4)$ and $240 \mathrm{kHz}(n=5)$ as shown in the insert in figure 10 . The amplitude of these peaks is very small, as observed in other tokamaks [11] corresponding to small radial displacements and density fluctuation levels. In ASDEX Upgrade a TAE radial displacement of around $0.3 \mathrm{~mm}$ was previously estimated [9]. In the analysis here the $\delta \varphi(f)$ for each TAE mode is obtained by integrating the phase spectrum over $10 \mathrm{kHz}$ around each peak and calculating the average value and standard deviation for a time window of $10 \mathrm{~ms}$. Figure 10 shows the phase spectrum and the radial profile of $\delta \varphi(f)$ for the $n=4$ TAE mode (solid line). The horizontal error bars indicate the uncertainty in the measurement of the density profile obtained from TS and LID over the $165 \mathrm{~ms}$ time interval considered. The maximum peak in $\delta \varphi(f)$ is around $\rho_{\text {pol }}=0.6 \pm 0.05$ with a less pronounced secondary peak at the edge.

The background fluctuation level at each radial position is also considered by integrating the phase spectrum over $10 \mathrm{kHz}$ at two frequency positions, just before the first peak and just after the last peak between $260 \mathrm{kHz}$ and $270 \mathrm{kHz}$, the average is calculated as well as the standard deviation $\sigma_{b g}$ using the technique described in [56]. The background fluctuation level (figure 10 - dashed line), while lower, is also found to roughly follow the mode amplitude. This could be due to either instrumental effects, such as interference in the core due to the shallower density gradient and the longer 
propagation distance between the density cutoff layer and the antenna, or to a real increase in the turbulence level around the TAE modes. Enhanced density turbulence associated with a strong TAE mode has previously been postulated by Kramer [57]. However, when the reflectometer data are displayed as quadrature plots $(I(t)$ signal plotted against the $Q(t)$ signal) a thin annulus is observed for the edge cutoff layers (indicative of strong reflection with little scattering) whereas core cutoff layers show a more circular cluster - which is indicative of strong scattering and interference effects.

The density fluctuation level can be calculated from the reflectometer phase signal using the 1D Geometric Optics model, however, note that the error bars in $\delta n_{e} / n_{e}$ are increased due to $L_{n}$. The $\delta n_{e} / n_{e}$ radial profile shows a similar shape as the reflectometer phase modulation profile with peak amplitudes of $\delta n_{e} / n_{e}=2.5 \times 10^{-4}(0.025 \%)$ at the edge and $\delta n_{e} / n_{e}=0.41 \times 10^{-4}(0.0041 \%)$ in the core, with respective density gradients of $2.5 \times 10^{20} \mathrm{~m}^{-4}$ and $0.48 \times 10^{20} \mathrm{~m}^{-4}$. These results suggest that the radial movement of the density cutoff is higher in the core although the relative density fluctuation level is lower than at the edge.

\subsection{Coherence and cross-phase analysis}

For the same discharge, \#21007, the coherence analysis technique is applied in order to compare different techniques for the determination of the radial structure of $n=4$ TAE mode. The coherence spectra between core reflectometry cutoff layer $(53 \mathrm{GHz})$ and the closest toroidally located Mirnov coil (labelled B31-13) is plotted in figure 11(a) and shows two clear peaks at $230 \mathrm{kHz}(n=4)$ and $240 \mathrm{kHz}(n=5)$. The significance level of the coherence is $\gamma_{0}=0.16$, defined by the number of spectral averages and window lengths, in this case $10 \mathrm{~ms}$ and $N_{a v}=39$. There are several Mirnov coils in the tokamak mid-plane at different toroidal locations. The cross-phase $\theta$ for the $n=4$ mode between each magnetic coil and reflectometer signal is calculated and the unwrap phase is plotted in figure 11(b) versus the toroidal angle $\phi$ for one radial position. $\phi=0$ corresponds to the toroidal position of frequency hopping reflectometer antennas. A linear regression is applied to the $\theta$ measurements. The slope of the line gives the toroidal mode number as well as the propagation direction of each TAE mode, while the offset $\theta_{0}$ indicates the value of $\theta$ at reflectometer position. At around $230 \mathrm{kHz}$ it is found that the mode has typically $n=4.0 \pm 0.12$ and propagates in the ion diamagnetic direction for both edge and core peak locations.

Figure 12(a) shows the coherence radial profiles for the $n=4$ and $n=5$ TAE modes using the adjacent B31-13 Mirnov coil measuring $B_{r}$. The coherence between the reflectometer and the other toroidally displaced Mirnov coils is generally very similar. The coils measuring $B_{\text {pol }}$ near the vessel surface have not been used as they give poor coherence. The coherence radial profile of course only gives an absolute value with no information on the sign of the radial displacement. This information is contained in the cross-phase spectra. The corresponding $\theta_{0}$ radial profile is shown in figure $12(\mathrm{~b})$ for the $n=4$ TAE mode. This plot essentially gives the radial phase behaviour of the 

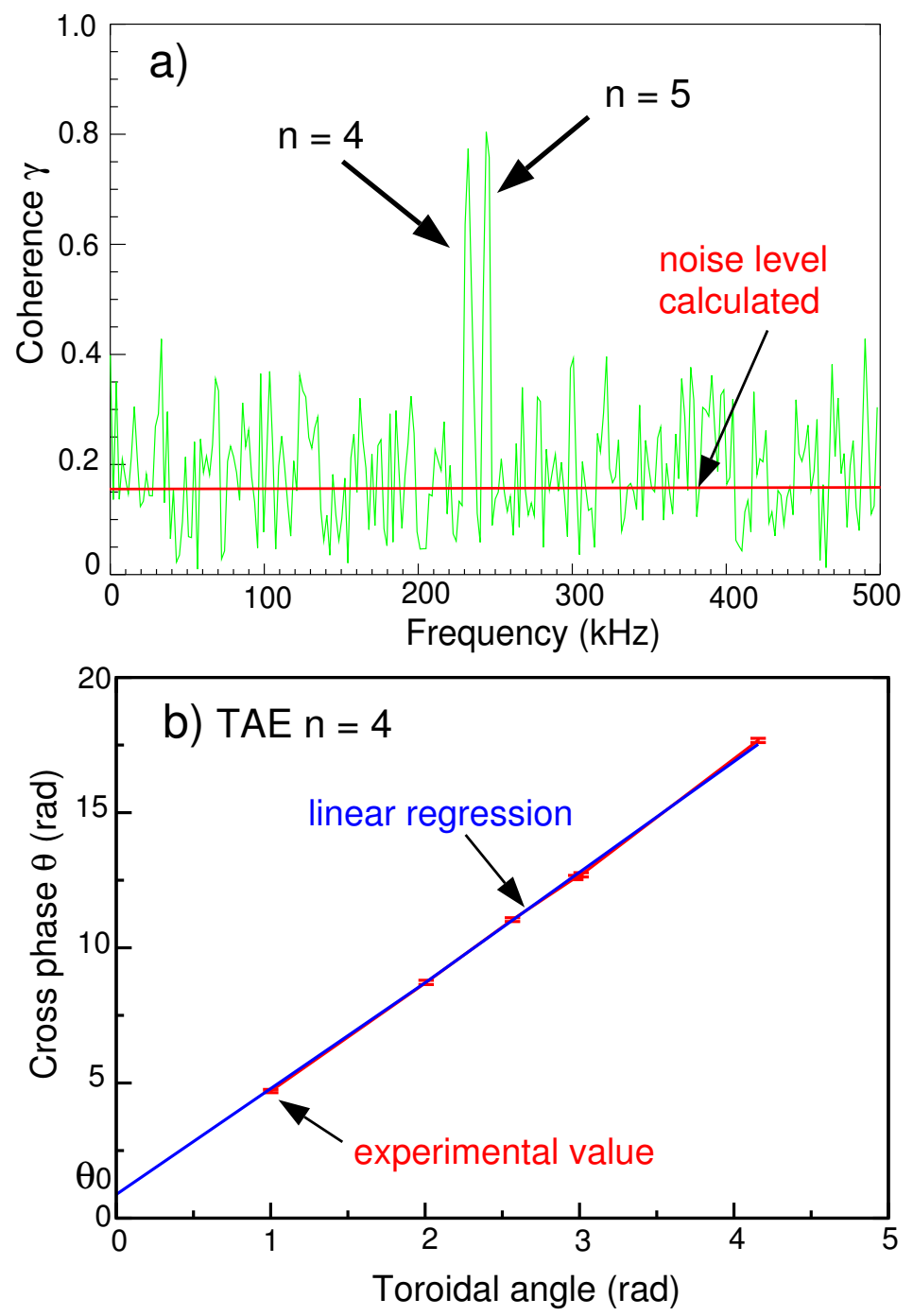

Figure 11. Coherence analysis: (a) Coherence between FLV-I (52GHz) and Mirnov coil B31-13 versus frequency, (b) Cross-phase versus toroidal angle between FLV-I (52GHz) and various Mirnov coils for shot \#21007.

mode. The profile for $n=5$ TAE mode is also similar. The $\theta_{0}$ is the cross-phase value corrected for the toroidal displacement between the reflectometer antenna and Mirnov coil positions and only values with a coherence greater than 0.4 are included in order to avoid noise effects. From the coherence and cross-phase profiles it is clear that an anti-node exists at $\rho_{\text {pol }}=0.8 \pm 0.02$ with a radial displacement that is out of phase with both the core and the very edge. The main contribution of the mode is localized in the core with a secondary peak at the edge. The maximum peak is around $\rho_{\text {pol }}=0.6$ and $\rho_{\text {pol }}=0.5$ for $n=4$ and $n=5$ TAE modes respectively. The radial structure appears to be consistent with the global TAE mode structure which has a radial width extending over a wide region. 

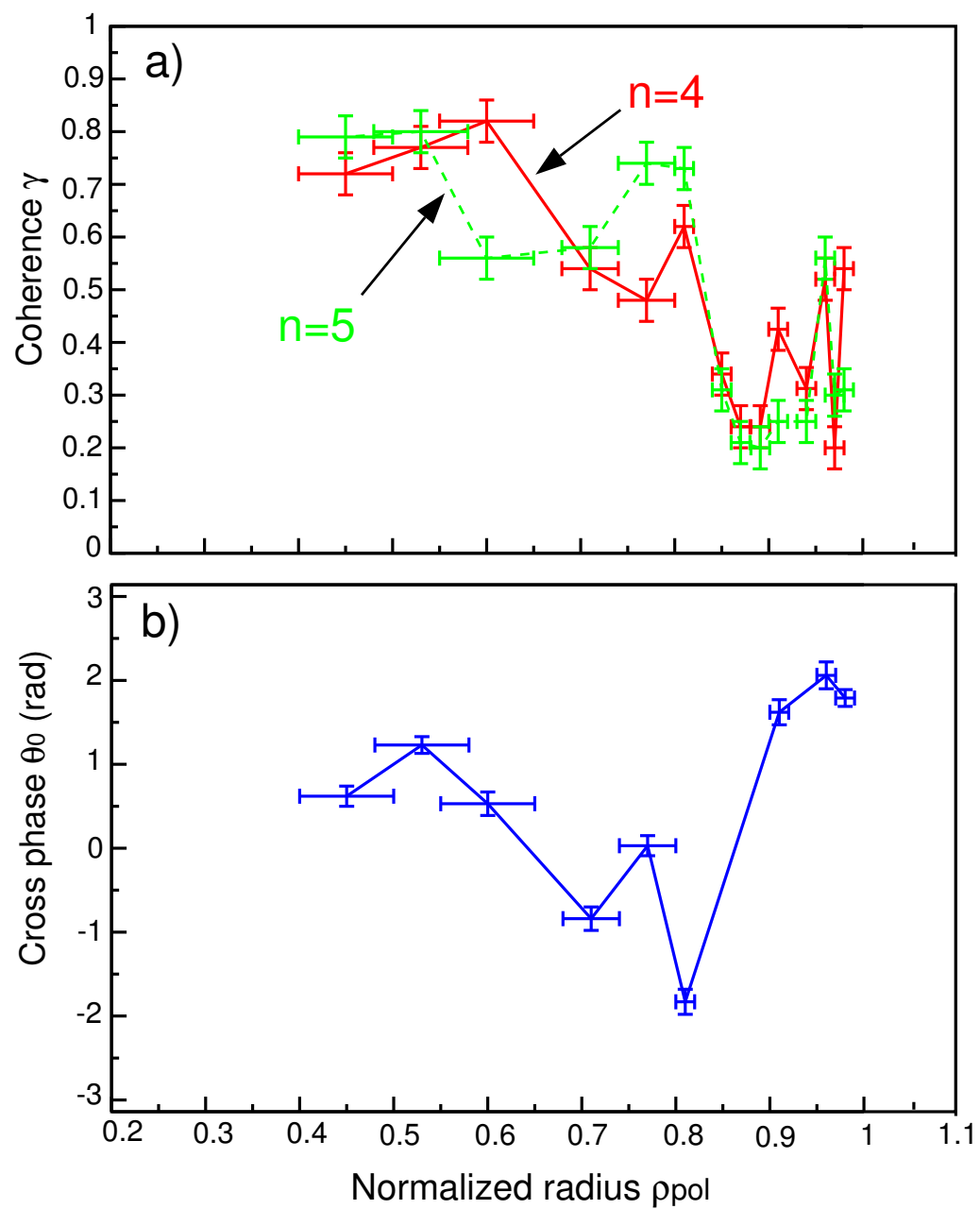

Figure 12. Coherence analysis: (a) Coherence profile for $n=4$ and $n=5$ TAE modes, (b) Cross-phase profile for $n=4$ TAE mode for shot \#21007.

\subsection{Simulations}

The comparison between numerical simulations and experimental results is an important aspect in studying fast particle mode behaviour, particularly for the validation of theories and thus the prediction of fast particle mode behaviour in ITER. Several simulation codes are available based on fluid or linear gyrokinetic models. For example, on the JET tokamak discrepancies between mode damping rates from simulation and experimental results were found and it was partly solved by using CASTOR-K code based on a hybrid MHD-gyrokinetic model [37]. The LIGKA code [19], developed at IPP, uses a self-consistent model including realistic fast particle orbits. This code shows damping rates close to the experimental ones [58] and appears to be due to the shape of the density profile at the plasma edge, i.e. for close (narrow) gaps the continuum damping is strong whereas for open (wide) gaps no significant mode conversion at the edge is found. The radial eigenfunction $\zeta_{0}(r)$ is of particular importance for comparison between simulation codes and experimental data. The LIGKA code normally gives the radial eigenfunction of 


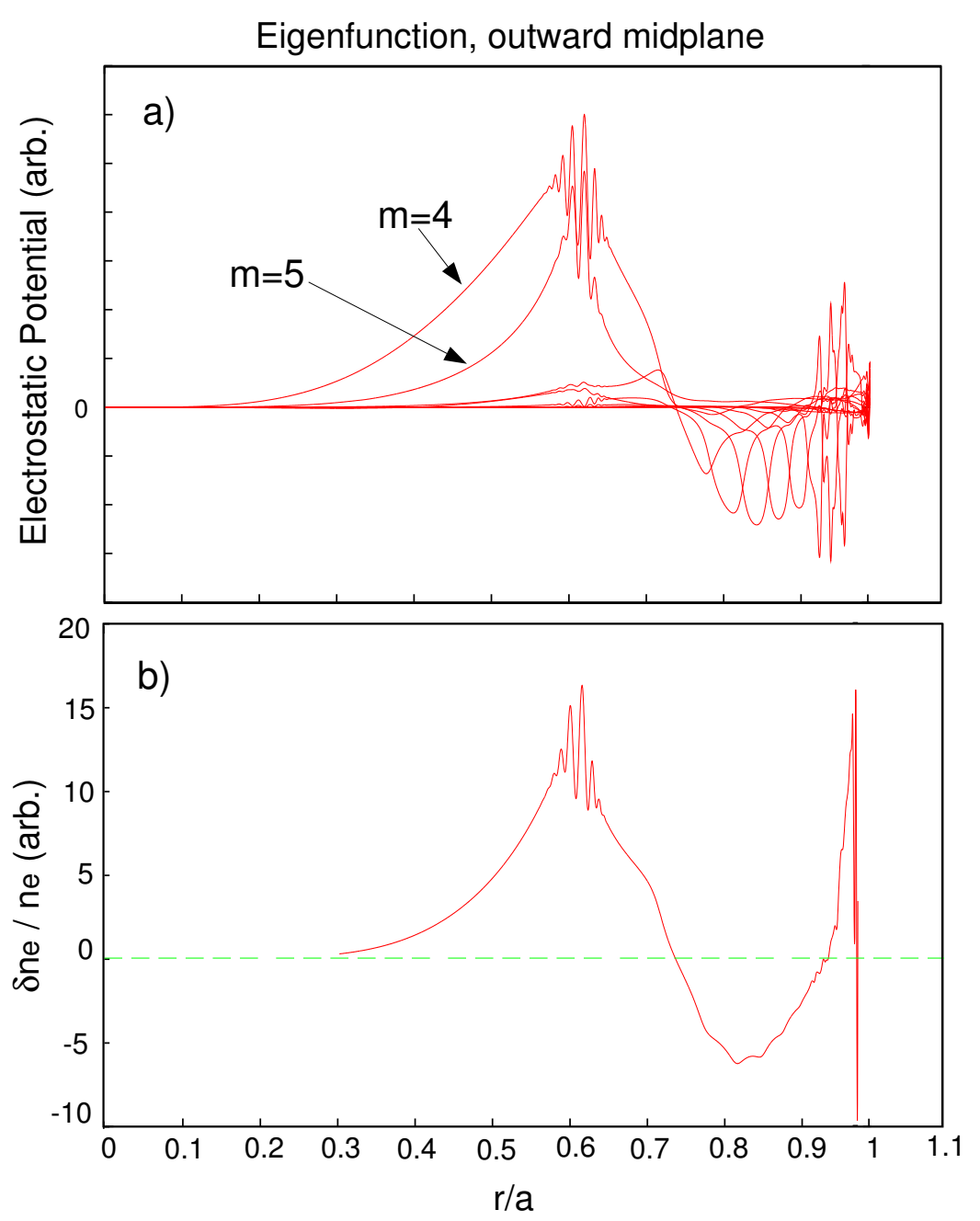

Figure 13. Simulated radial eigenfunction of (a) electrostatic potential and (b) density fluctuations for $n=4$ TAE from the LIGKA code for shot \#21007.

the electrostatic/electromagnetic potential, but recently it has been extended to give the radial eigenfunction of the corresponding density fluctuations $\delta n_{e} / n_{e}$ - which of course depends on the density gradient profile. The perturbed density is calculated kinetically by integrating over the velocity space and summing up over all the fourier harmonics of the eigenfunctions for the perturbed electrostatic and electromagnetic potential.

In figure 13 the different poloidal harmonics of the electrostatic potential and the envelope of the density fluctuation radial eigenfunction are plotted, which can be compared with the experimental results in figure 12. The agreement between simulation and the reflectometer/Mirnov-coil coherence and cross-phase analysis is in general very good. The relative amplitude as well as the sign of the radial eigenfunction are reproduced. The reflectometer phase magnitude profile in figure 10 is also in good agreement with the LIGKA simulation, although unfortunately no information on the sign of the radial eigenfunction is available from the phase perturbation technique. Nevertheless, both analysis techniques show a broader radial extent of $n=4$ TAE 


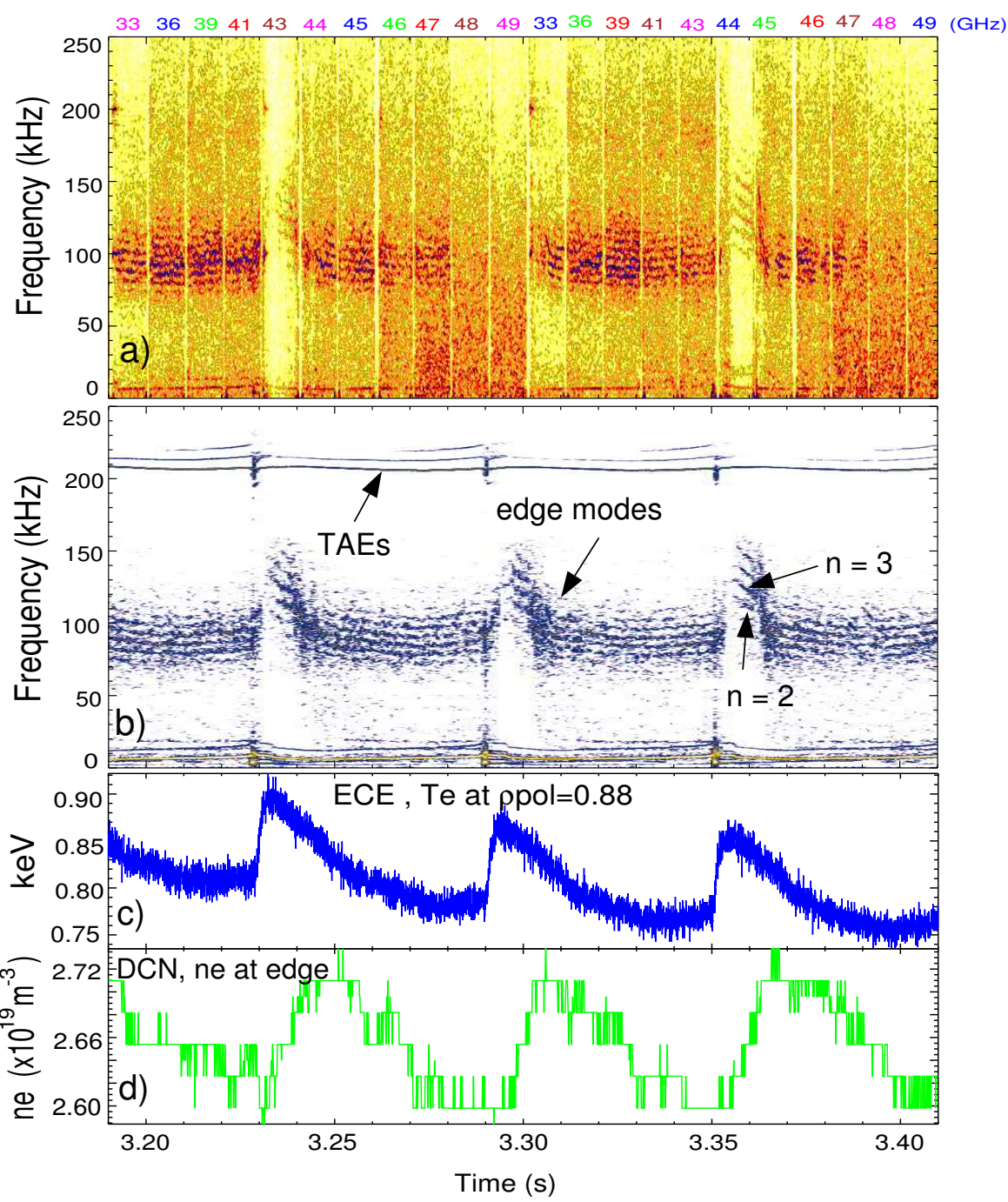

Figure 14. Time-frequency spectrograms of (a) FLQ-I stepped frequency reflectometer, (b) Mirnov coil, with time traces of (c) edge $T_{e}$ and (d) edge line averaged $n_{e}$ for shot \#21011.

than predicted by the LIGKA code. But, it is clear from both the simulation and the experimental data that two distinct maxima are present, one at the edge and one in the core. Further, the comparison with the LIGKA code also suggests that the rapid oscillations present in the experimental radial eigenfunction at the edge are due to nonideal effects, indicating a closed gap [59].

\section{Edge modes}

\subsection{Characteristics of edge modes}

Edge MHD modes rotating in the electron diamagnetic drift direction with toroidal mode numbers $n=2,3,4$ are observed, with and without TAEs, when ICRH power exceeds $3 \mathrm{MW}$. Figure 14 shows spectrograms of the Q-band reflectometer and Mirnov 
coil signals together with time traces of edge temperature $T_{e}$ and density $n_{e}$ for shot \#21011. The presence of sawteeth is clearly seen in both the edge $n_{e}$ and $T_{e}$. A dramatic decrease in the overall density fluctuation level can be seen in the reflectometer spectrum just after each sawtooth (irrespective of probing frequency). This could be due to either a change on the local density gradient $\nabla n_{e}$, a reduction in the fluctuation level or a combination of both. Unfortunately, fast time resolved density profiles are not available so an investigation of $\nabla n_{e}$ just after a sawtooth crash can not be made. Both spectrograms show a rapid jump in the frequency of the edge modes within $2 \mathrm{~ms}$ after each sawtooth crash. This behaviour can be interpreted as a direct scaling of the frequency of edge modes with the local temperature $T_{e}$ and density $n_{e}$. After the increase of the edge $T_{e}$, the frequency difference between two consecutive toroidal mode numbers increases by around $4 \mathrm{kHz}$ which suggests an increase in the plasma rotation. Unfortunately the effect of sawtooth crashes on the plasma rotation can not be confirmed by the Charge Exchange Spectroscopy diagnostics due to the absence of neutral beam injection in these pure ICRH heated shots.

\subsection{High and Low field side comparison}

Figure 15 shows a series of power spectra from the high (HFS) and low field side (LFS) reflectometer channels at three different radial positions close to the plasma edge (homodyne signals from the multi-channel profile reflectometer operating with same fixed launch frequencies for LFS and HFS) for shot \#20204. The probing frequency is the same for HFS and LFS, it was considered that the density is constant along the flux surfaces. In this discharge TAE modes with $n=4$ and 5 are observed in the plasma edge in the HFS, but not in LFS (peaks around $200 \mathrm{kHz}$ starting near the separatrix). On the LFS there is a mode around $80-90 \mathrm{kHz}$, starting again near the separatrix but growing to become the dominant feature at $\rho_{\text {pol }} \simeq 0.97$. At this radius it is dominant in both the low and high field sides. Further, a second harmonic of this mode with lower amplitude also appears in both the HFS and LFS. This feature is also confirmed by the Q-band frequency hopping reflectometer in figure 16(a) with a cutoff layer at a similar radial position. Figure 16(b) shows less pronounced edge modes toward the plasma interior. The absence of the lower frequency edge mode at $\rho_{\text {pol }} \simeq 0.99$ in the HFS spectra may be due to different radial mode structures in the HFS and LFS. Unfortunately due to the homodyne nature of the signals from the FM-CW profile reflectometer no further quantitative analysis can be made for the HFS.

The power spectra also give information about the background density turbulence. Generally the fluctuation spectra are characterized by a flat spectral power at low frequencies extending up to a knee point and then decaying with a constant spectral index. The frequency of the spectral knee point scales with the tranverse plasma rotation velocity [60]. On both high and low field sides no significant frequency shift is observed indicating only a small variation in the poloidal rotation - as may be expected in L-mode discharges [61]. For the three radial positions the spectral index is practically constant 

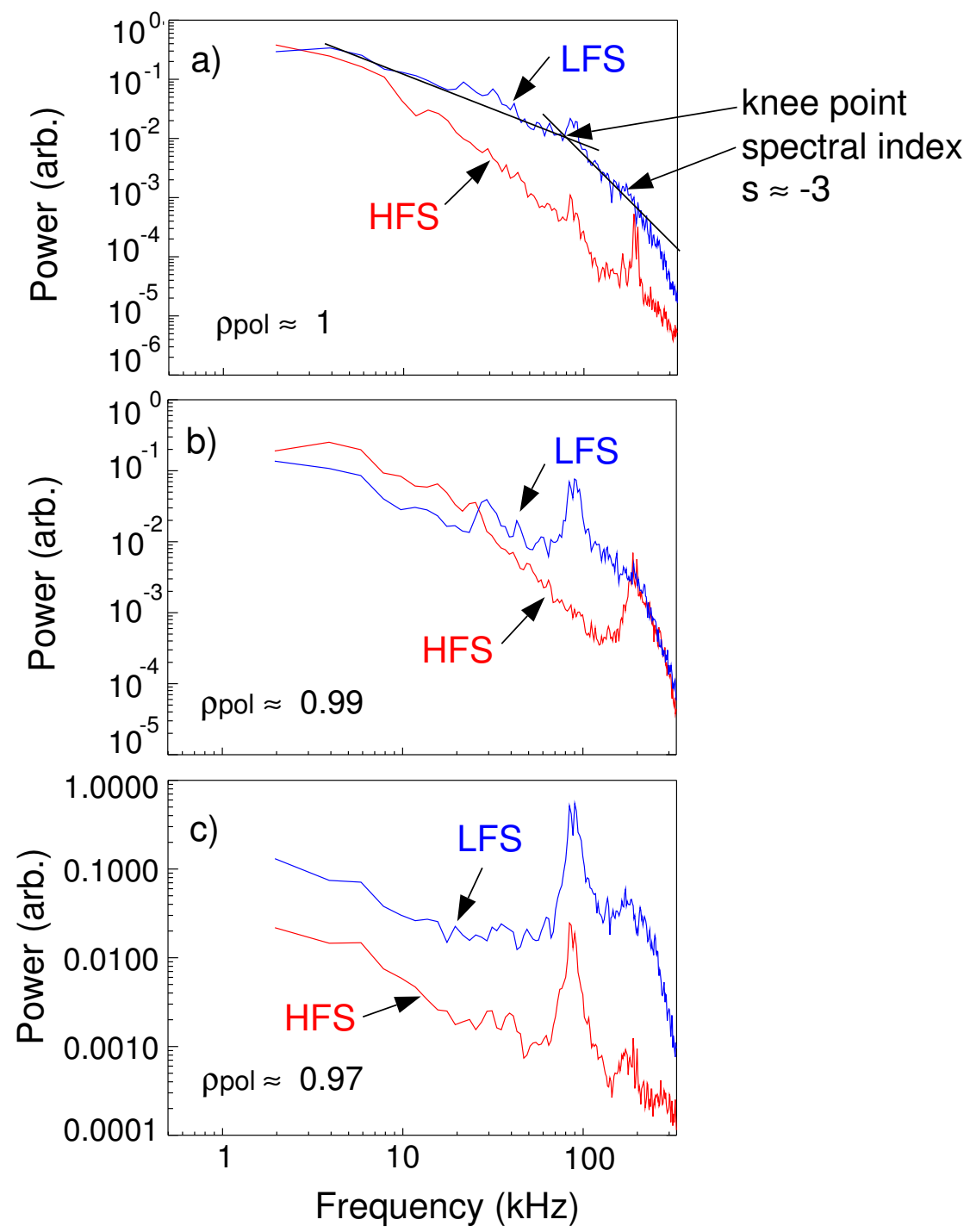

Figure 15. Reflectometer power spectra from high and low field sides at (a) near the separatrix, (b) $\rho_{\text {pol }} \simeq 0.99$ and (c) $\rho_{\text {pol }} \simeq 0.97$ for shot \#20204

indicating that the fluctuation wavenumber spectra is the same.

\subsection{Radial structure}

The reflectometer spectrograms in figures 7 and 14 for shots \#20489 and \#21011 show the presence of MHD modes in the plasma edge. Unfortunately for shot \#21011 the density profile is rather flat inhibiting core cutoff localization with the O-mode reflectometers. Nevertheless, the edge modes are observed in the V-band frequency hopping channel even though the probing frequencies are above the maximum density cutoff. Here the diagnostic is operating in interferometry/refractometry mode, which, although lacking signal localization, is compensated by an enhanced phase sensitivity to fluctuations [48]. The edge localization of these modes is, however, confirmed by the SXR cameras - with their good sensitivity to the core, where the modes are not observed 

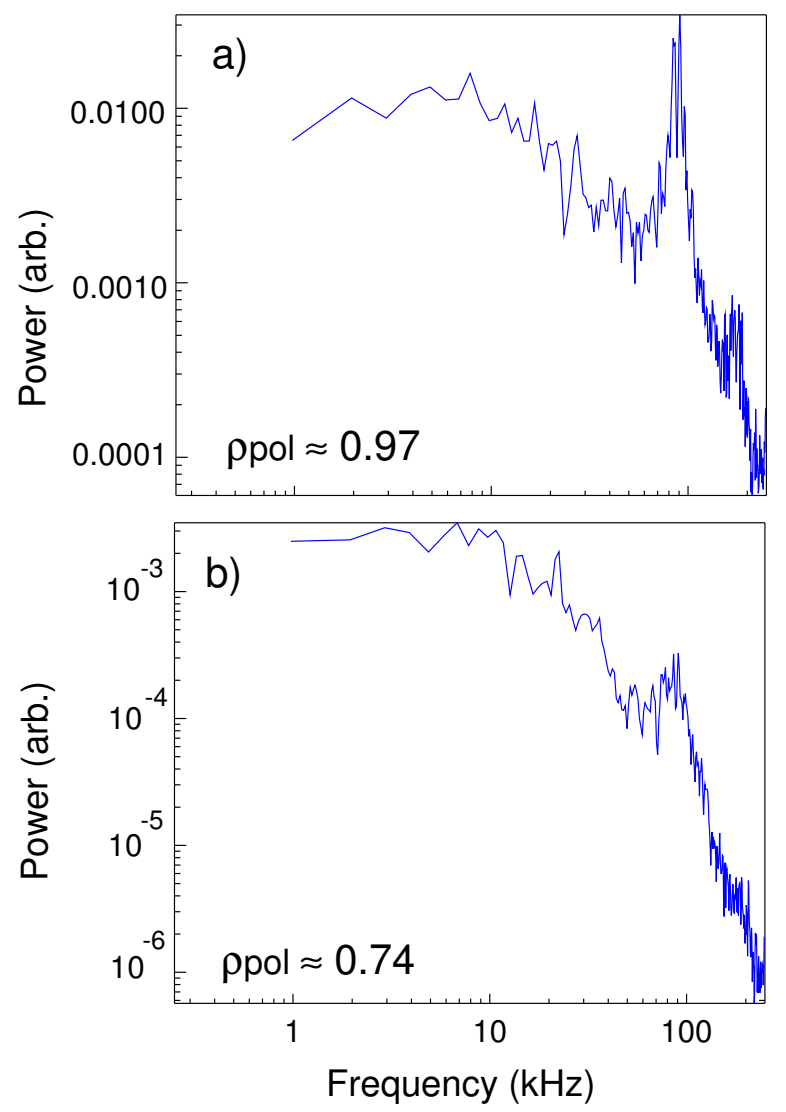

Figure 16. Reflectometer power spectra from low field side with FLQ-I and FLV-I (a) $\rho_{\text {pol }} \simeq 0.97$ and (b) $\rho_{\text {pol }} \simeq 0.74$ for shot \#20204

- and by the edge ECE diagnostic, where they are seen [62].

Although the radial reach of the reflectometers is limited for shot \#21011, a radial structure analysis was performed and the results presented in figure 17 using (a) the coherence and (b) the reflectometer phase perturbation techniques. For the coherence technique the significance level of the coherence is $\gamma_{o} \approx 0.21$ (6 ms window length and $N_{a v}=22$ ). Using an FFT window of 512 points leads to a cluster of several ill-defined spectral peaks. But, increasing the number of FFT points to resolve the peaks reduces the number of spectral ensemble averages which unacceptably raises the coherence significance threshold. However, since the coherence value of each spectral peak is similar, a reduced resolution is accepted and the highest coherence peak value is taken.

For the reflectometer phase perturbation analysis, since the edge mode amplitude is particularly large (in contrast to the TAE situation), as shown in the reflectometer phase spectrum in figure 17 (b - insert) where the background fluctuation level either side of the edge mode cluster is small compared to their phase magnitude, instead of using the phase spectrum magnitude the raw phase signal is pass-band filtered $(65-135 \mathrm{kHz}$ for all probing frequencies except for $43 \mathrm{GHz}$ with $80-150 \mathrm{kHz}$ ). For strong, dominant modes this method is more effective for isolating the turbulence background from the 


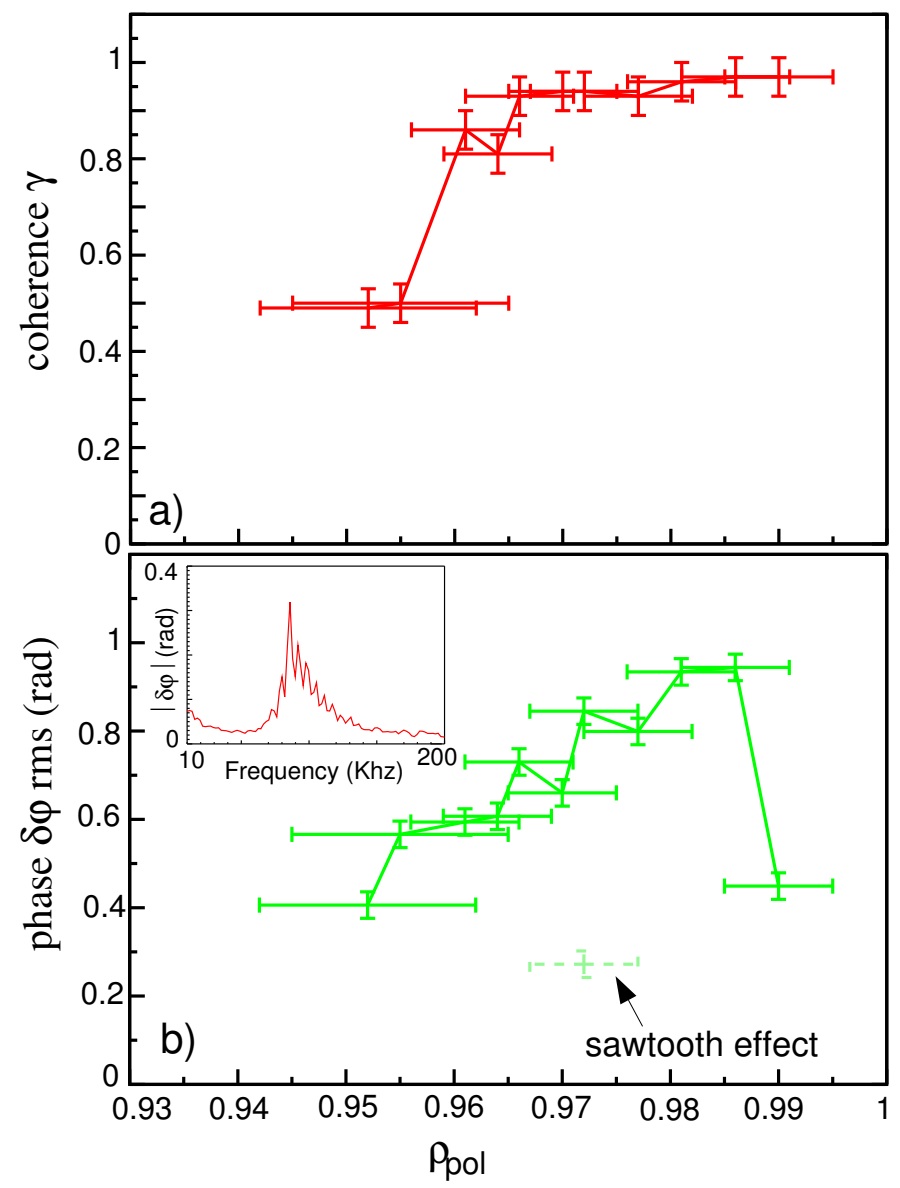

Figure 17. Edge modes radial profiles using (a) reflectometer-Mirnov coherence and (b) reflectometer phase modulation (rms) analysis for shot \#21011 over several sweeps.

modes. The phase RMS is then calculated from the filtered signal to give the radial profile in figure $17(\mathrm{~b})$.

Overall, the two techniques give consistent results and indicate that the modes are stronger in the pedestal region with a decaying envelope of coherence and phase RMS values toward the plasma interior. The coherence radial profile is broader than phase RMS profile, as observed in the previous section for the TAE behaviour. At $\rho_{\text {pol }} \simeq 0.97$ (and 0.99) the phase RMS is observed to drop but with no corresponding significant reduction in the coherence value. After a detailed study of different reflectometer sweeps it is concluded that the drop at $\rho_{\text {pol }} \simeq 0.97$ is a temporal feature due to the effect of the sawtooth, while the dip at $\rho_{\text {pol }} \simeq 0.99$ appears to be a localized reduction in $\delta n_{e} / n_{e}$.

\section{Alfvén Cascades}

In the JET tokamak, Alfvén Cascades (ACs) are regularly observed during the plasma current ramp-up when ICRH and lower hybrid heating/current drive (LHCD) are applied in order to create a non-monotonic $q(r)$ profile and trigger an ITB [47]. In Alcator C-mod, ACs are also observed during the current rise with strong ICRF heating 


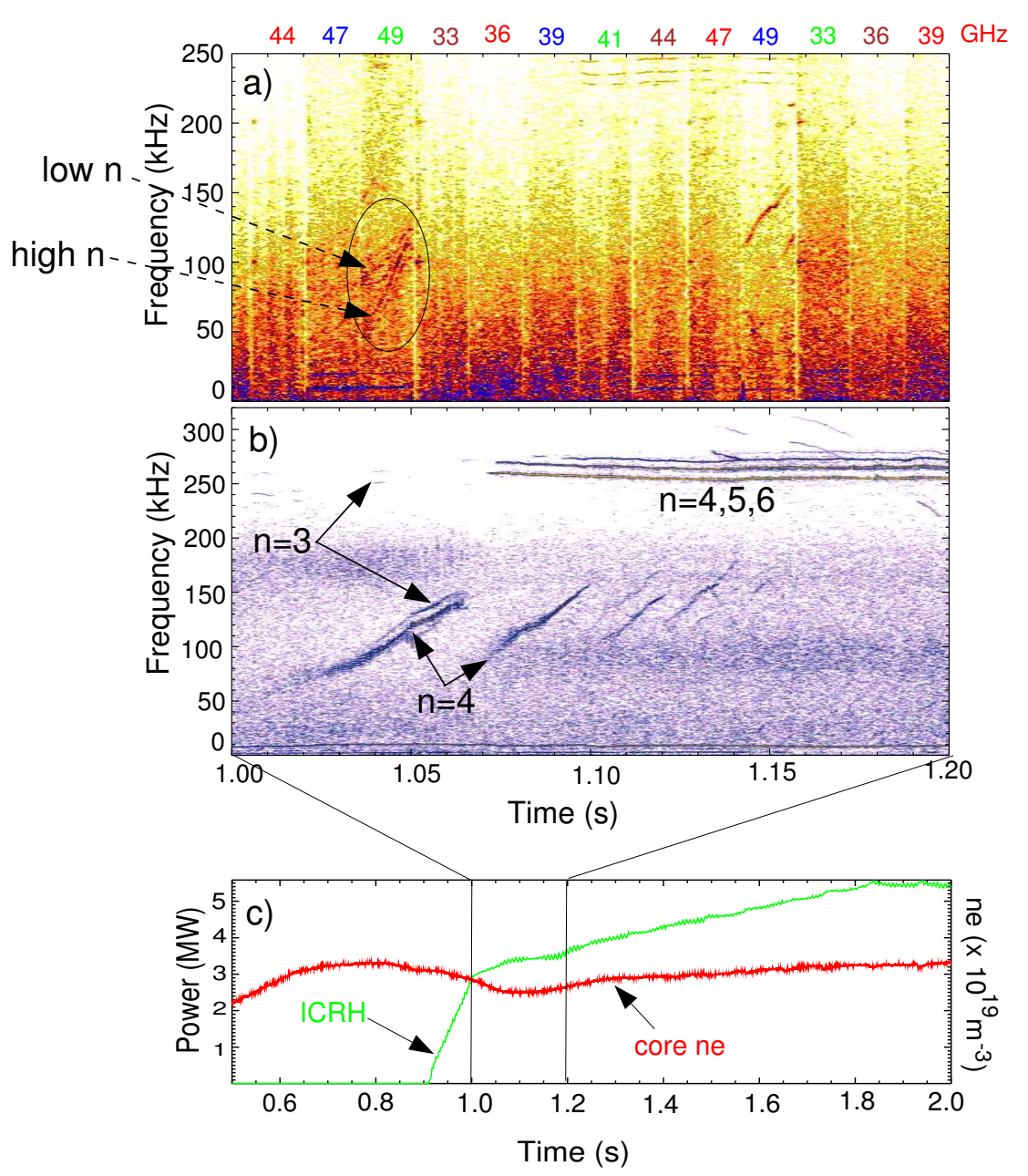

Figure 18. Shot \#20398: (a) FLQ-I spectrogram, (b) Magnetic spectrogram and (c) Time traces of ICRH and $n_{e}$.

[18]. In ASDEX Upgrade, modes with upward frequency sweeping are likewise observed in the early phase of some discharges with ICRH power ramp-up and $I_{p}$ constant. For example, in shot \#20398 both magnetic and reflectometry data exhibit these modes between $1-1.2 \mathrm{~s}$, as shown in the spectrograms in figure 18. After $1.07 \mathrm{~s}$ both TAEs and these chirping modes coexist. At $t=1.32 \mathrm{~s}$ a sawteeth crash occurs, indicating that $q_{\text {min }}$ is decreasing in time.

As often stated, internal measurements can offer better frequency and time resolution than external magnetic pick-up coils. In JET, ACs were clearly observed using the O-mode reflectometer diagnostic, but, due to the flat core density profile they were operating in the interferometry/refractometry mode with the probing wave reflected from the inner wall of the torus [48]. Hence no information about the localization of the ACs was possible. However, in ASDEX Upgrade for shot \#20398 the density profile was reasonably steep thus allowing the O-mode reflectometry diagnostic good radial coverage and thus localization of the ACs. From magnetic measurements the toroidal mode numbers can be deduced. After 1.07 s $n=4,5,6,7$ TAEs exist (around 
$250-270 \mathrm{kHz}$ ), while the chirping modes start at around $50 \mathrm{kHz}$ and have toroidal numbers of $n=3,4$ and rotate in the ion diamagnetic drift direction, like the TAEs. For this shot the fast frequency hopping reflectometers were configured with a data sample rate of $500 \mathrm{kHz}$, which should permit the discrimination of MHD modes only up to $250 \mathrm{kHz}$, however, as the hardware anti-aliasing analog filters do not have a sharp edge frequency response, it is possible in this case to use the aliasing effect to allow the estimation of the radial extent of TAE modes, $\rho_{\text {pol }} \approx 0.35-0.68( \pm 0.05)$ using Thomson scattering density profiles. The radial extent of the TAEs are in good agreement with the results from CASTOR code at $t=1.76 \mathrm{~s}$ as shown in figure 4 . Combining the $\mathrm{Q}$ and V-band reflectometer measurements, the chirping modes are core localized with $\rho_{\text {pol }} \approx 0.2-0.4( \pm 0.05)$.

In the reflectometry data (around $1.04 \mathrm{~s}$ in figure 18, $49 \mathrm{GHz}$ probing frequency) more frequency sweeping discrete modes can be seen than in the magnetic data. It is known that modes with higher toroidal mode numbers exhibit a more rapid frequency sweeping, and the higher $n$ modes re-occur more often than the lower $n$ modes [45]. As observed in JET, in this shot at $1.04 \mathrm{~s}$ the magnetic coils detect only the lower $n$ modes while the reflectometers see additional higher $n$ mode number with more rapid frequency sweeping. The slope of the frequency sweeping is deduced from reflectometry measurements and is approximatively $23 \times 10^{5} \mathrm{~s}^{-2}$ and $41 \times 10^{5} \mathrm{~s}^{-2}$ for lower and higher $n$ modes respectively. In both the magnetic and reflectometry measurements the ACs rise until around $150 \mathrm{kHz}$ and stop before the TAE frequency. This behaviour may be explained by the effect of the pressure gradient on the ACs [50]. The slope of the frequency sweeping also varies in time, which is not common in standard ACs observed in other tokamaks.

Theoretical and simulation studies have been made for the ACs observed in ASDEX Upgrade $[63,64]$. The starting frequency of these chirping modes is below the geodesic frequency - considered the lowest limit for conventional ACs. Furthermore, the $n=4$ $\mathrm{AC}$ and TAE are present at the same time suggesting that these ACs are non-standard ACs possibly created by the coupling to the sound branches.

\section{Conclusion and discussion}

The combination of fast frequency hopping reflectometers and magnetic measurements has allowed the detailed measurement of the radial structure of core and edge localized MHD modes in ASDEX Upgrade. The radial eigenfunction of a mode is of particular importance in allowing detailed comparisons of experimental with simulation results, from codes such as CASTOR, LIGKA and MISHKA [65] codes, in order to fully characterize instabilities. In particular, it is crucial for a detailed understanding of the dissipative mechanism that damp fast particle driven Alfvén Eigenmodes.

The frequency hopping reflectometer uses a fixed pre-programmed launch frequency pattern repeated throughout the discharge. In shots with almost constant plasma parameters, in particular density, the diagnostic gives highly reproducible results. For 
example, the same radial position and extent of instabilities are obtained from successive frequency sweeps. In fairly low density discharges, i.e. $\bar{n}_{e}=3-4.10^{19} \mathrm{~m}^{-3}$ with a moderately steep core density gradient the reflectometer system can cover almost the complete plasma core to edge region. These conditions are ideal for studying fast particle modes such as TAEs and ACs.

The radial eigenfunctions of MHD and fast particle modes were obtained using two different analysis techniques: coherence between reflectometry and magnetic signals, and the reflectometer phase perturbations induced by these modes. The coherence technique must combine high frequency resolution with a low statistical significance level, so, for the fixed probing step-lengths necessary for a reasonable temporal resolution, there is a compromise on the choice in the optimum number of FFT points. Regarding the reflectometer phase perturbation analysis, two approaches can be used: phase spectral magnitude and raw phase RMS with band-pass filtering. The first approach allows one to obtain the radial structure for different toroidal mode numbers, but for small spectral peak amplitude the background fluctuation level must be taken into account. On the other hand, the phase RMS is the more usual technique applicable for low turbulence backgrounds. Normally this value is used to calculate density fluctuation level using a 1D Geometric Optics model. However, due to the filter restrictions it is not possible to extract the phase RMS value for different modes with small frequency separation.

These two techniques were applied to the analysis of TAEs and edge MHD modes. For one particular discharge, shot \#21007, radial eigenfunction of a $n=4$ TAE was obtained using coherence and cross-phase profiles giving the mode amplitude and sign respectively. For comparison, the radial profile was obtained from the reflectometer phase perturbation analysis, but without giving the sign of the radial eigenfunction.

There is good agreement between the simulated radial eigenfunction from the LIGKA code and the experimental one. In this discharge, the LIGKA code predicts continuum damping at the edge which is characteristic of closed gaps. The ratio of the density fluctuation level between the edge and core peaks is of the order of 2 in the simulation, whereas experimental results give a ratio closer to 5 . This discrepancy could arise from one of several sources. The translation of the potential (in the simulation) or the reflectometer phase (in the experiment) to $\delta n_{e}$ is very sensitive to the density gradient. Particularly in the steep plasma edge the measured gradient is subject to large errors. Additionally, the 1D Geometric Optics model used to convert the reflectometer phase does not consider for example the radial fluctuation wavelength of the mode, the wavefront curvature, or the geometry of antennas [29].

The radial structures obtained with the coherence and phase perturbation analysis techniques are in good agreement for both edge and core. In fact, the phase perturbation analysis indicates the physical amplitude of radial cutoff layer movement whereas the coherence technique shows the relative strength between different peaks. For this, the two approaches can be seen as complementary. For the $n=4$ TAE radial eigenfunction, comparing the ratio between the core and edge peaks, gives a value of around 1.7 using the phase perturbation approach and 1.6 from the coherence analysis. For the edge 
modes the radial structure gives a ratio between the peak and the last value of 2.33 and 2.0 for the phase perturbation and the coherence techniques respectively. This excellent agreement is a good validation of the analysis procedures.

The edge MHD modes have not yet been identified with certainty, although several possibilities can be considered. Their characteristics (edge localization, rotation in the electron diamagnetic drift direction with $n=2,3,4$ toroidal mode numbers) lead to at least two candidates: Washboard modes (WBs) [66] or edge localized TAE [67, 62]. In the first case, WBs are observed in H-mode discharges with type I ELMs, but only L-mode discharges are considered in the present study. On the other hand, to be a TAE requires confirmation of the Alfvénic character of these modes, that is, the mode frequency must vary appropriately with the local plasma density and with $B_{t}$.

\section{Acknowledgments}

We wish to especially thank L.Meneses and A.Ferreira for assistance with the reflectometer hardware, R.Merkle for assistance with the data acquisition hardware, B.Kurzan for validation of Thomson scattering data and V.Igochine for fruitful discussions on SXR data.

This work has been carried out within the framework of the Contract of Association between the European Atomic Energy Community and Instituto Superior Técnico. Financial support was also received from Fundação para a Ciência Tecnologia and Programa Operacional Ciência, Tecnologia e Inovação do Quadro Communitário de Apoio III.

\section{References}

[1] ITER Physics Expert Group on Energetic Particles, Heating and Current Drive et al. 1999 Nucl. Fusion 392471

[2] Wong K L et al. 1991 Phys. Rev. Lett. 661874

[3] Ishikawa M et al. 2006 Nucl. Fusion 46 S898

[4] Saigusa M et al 1995 Plasma Phys. Control. Fusion 37295

[5] Carolipio E M et al. 2001 Phys. Plasmas 83391

[6] Fredrickson E D et al. 2006 Phys. Plasmas 13056109

[7] Snipes J A et al. 2006 Nucl. Fusion 461036

[8] Fasoli A et al 1997 Plasma Phys. Control. Fusion 39 B287

[9] Borba D et al. 2004 Plasma Phys. Control. Fusion 46809

[10] Durst R D et al. 1992 Phys. Fluids B 43707

[11] Nazikian R et al. 1998 Phys. Plasmas 51703

[12] Nazikian R et al. 2003 Phys. Rev. Lett. 91125003

[13] Sabot R et al 2006 Plasma Phys. Control. Fusion 48 B421

[14] Udintsev V S et al 2006 Plasma Phys. Control. Fusion 48 L33

[15] Van Zeeland M A et al. 2006 Phys. Rev. Lett. 97 135001-1

[16] Van Zeeland M A et al 2005 Plasma Phys. Control. Fusion 47 L31

[17] Van Zeeland M A et al 2006 Nucl. Fusion 46 S880

[18] Snipes J A et al. 2005 Phys. Plasmas 120516102 
[19] Lauber Ph 2003 Linear Gyrokinetic Description of Fast Particle Effects on the MHD Stability in Tokamaks (Ph.D. thesis TU Munchen)

[20] Cupido L et al. 2006 Rev. Sci. Instrum. 77 10E915

[21] Silva A et al. 1996 Rev. Sci. Instrum. 674138

[22] Nave M F F et al. 2004 Rev. Sci. Instrum. 754274

[23] Kramer-Flecken A et al. 2004 Nucl. Fusion 441143

[24] Schirmer J et al. 2004 31st EPS Conf. Cont. Fusion Plasma Phys. ECA vol. 28G, P4.127

[25] Bendat J S and Piersol A G 1986 Random data: Analysis and Measurement Procedures (New York: Wiley)

[26] Conway G D and Elliott J A 1987 J. Phys. E: Sci. Instrum. 201341

[27] Estrada T et al. 2001 Phys. Plasmas 82657

[28] Conway G D et al. 1996 Plasma Phys. Control. Fusion 38451

[29] Nazikian R et al. 2001 Phys. Plasmas 81840

[30] Fanack C et al. 1996 Plasma Phys. Control. Fusion 381915

[31] Bretz N 1992 Phys. Fluids B 4 (8) 2414

[32] Bretz N 1997 Rev. Sci. Intrum. 682927

[33] Wang G et al. 2006 Nucl. Fusion 46 S708

[34] Seo S-H et al. 2005 Rev. Sci. Intrum. 76036104

[35] Klüber O et al. 1991 Nucl. Fusion 31907

[36] Cheng C Z et al. 1986 Phys. Fluids 293695

[37] Borba D et al. 2002 Nucl. Fusion 421029

[38] Kerner W et al. 1998 J. Comp. Phys. 142271

[39] Fu G Y et al. 1995 Phys. Rev. Lett. 752336

[40] Berk H L et al. 1995 Phys. Plasmas 23406

[41] Kramer G J et al. 2004 Phys. Rev. Lett. 92 015001-1

[42] Wong King-Lap 1999 Plasma Phys. Control. Fusion 41 R1

[43] Mett R R et al. 1992 Phys. Fluids B 42885

[44] Kimura H 1998 Nucl. Fusion 381303

[45] Berk H L et al. 2001 Phys. Rev. Lett. 87) 185002

[46] Breizman B N et al. 2003 Phys. Plasmas 103649

[47] Sharapov S E et al. 2002 Phys. Plasmas 92027

[48] Sharapov S E et al. 2004 Phys. Rev. Lett. 93165001

[49] Breizman B N et al. 2005 Phys. Plasmas 12 112506-1

[50] Fu G Y et al. 2006 Phys. Plasmas 13052502

[51] Lauber $\mathrm{Ph}$ et al. 2007 York

[52] Heidbrink W W et al. 1999 Phys. Plasmas 61147

[53] Chu M S et al. 1992 Phys. Fluids B 43713

[54] Wootton et al. 1990 Phys. Fluids B 22879

[55] Fasoli A et al. 1998 Phys. Rev. Lett. 815564

[56] Bevington P R and Robinson D K 2003 Data reduction and error analysis for the physical sciences p. 42, 43 (Mc Graw-Hill)

[57] Kramer G J et al. 2006 48th Annual Meeting of the Division of Plasma Physics, APS NP1.00036

[58] Lauber $\mathrm{Ph}$ et al. 2005 Phys. Plasmas 12122501

[59] Guenter S et al. 2006 submitted to Nucl. Fusion

[60] Conway G D et al. 2002 Plasma Phys. Control. Fusion 441167

[61] Conway G D et al. 2004 Plasma Phys. Control. Fusion 46951

[62] Sassenberg K et al. to be submitted

[63] Brüdgam M 2006 Numerische Untersuchung der Alfvénkaskaden und deren Existenzkriterien (Diploma thesis University Rostock)

[64] Lauber Ph et al. 2006 AIP Conf. Proc. 871147

[65] Mikhailovskii A B et al. 1997 Plasma Phys. Rep. 23844 
[66] Perez C P et al. 2004 Plasma Phys. Control. Fusion 4461

[67] Maraschek M et al. 1997 Phys. Rev. Lett. 794186 